Seton Hall University eRepository@Seton Hall

Seton Hall University Dissertations and Theses (ETDs)

Seton Hall University Dissertations and Theses

Spring 5-19-2018

\title{
The Immediate and Long-lasting Cognitive Consequences of Adolescent Chronic Sleep Restriction
}

Kerry Howard

kerry.howard@student.shu.edu

Follow this and additional works at: https://scholarship.shu.edu/dissertations

Part of the Biological Psychology Commons, Cognitive Psychology Commons, and the Social Psychology Commons

\section{Recommended Citation}

Howard, Kerry, "The Immediate and Long-lasting Cognitive Consequences of Adolescent Chronic Sleep Restriction" (2018). Seton Hall University Dissertations and Theses (ETDs). 2526.

https://scholarship.shu.edu/dissertations/2526 


\title{
THE IMMEDIATE AND LONG-LASTING COGNITIVE CONSEQUENCES OF ADOLESCENT CHRONIC SLEEP RESTRICTION
}

\author{
by \\ Kerry Howard
}

A Thesis Submitted In Partial Fulfillment of the Requirements for the Master of Science in Experimental Psychology-Thesis with a Concentration in Cognitive Neuroscience

The Department of Psychology

Seton Hall University

May 2018 
(C) 2018 Kerry Howard 


\section{SETON HALL UNIVERSITY \\ College of Arts \& Sciences}

\section{APPROVAL FOR SUCCESSFUL DEFENSE}

Masters Candidate, Kerry Howard, has successfully defended and made the required modifications to the text of the master's thesis for the M.S. during this Spring Semester 2018.

\section{THESIS COMMITTEE}

(please sign and date beside your name)

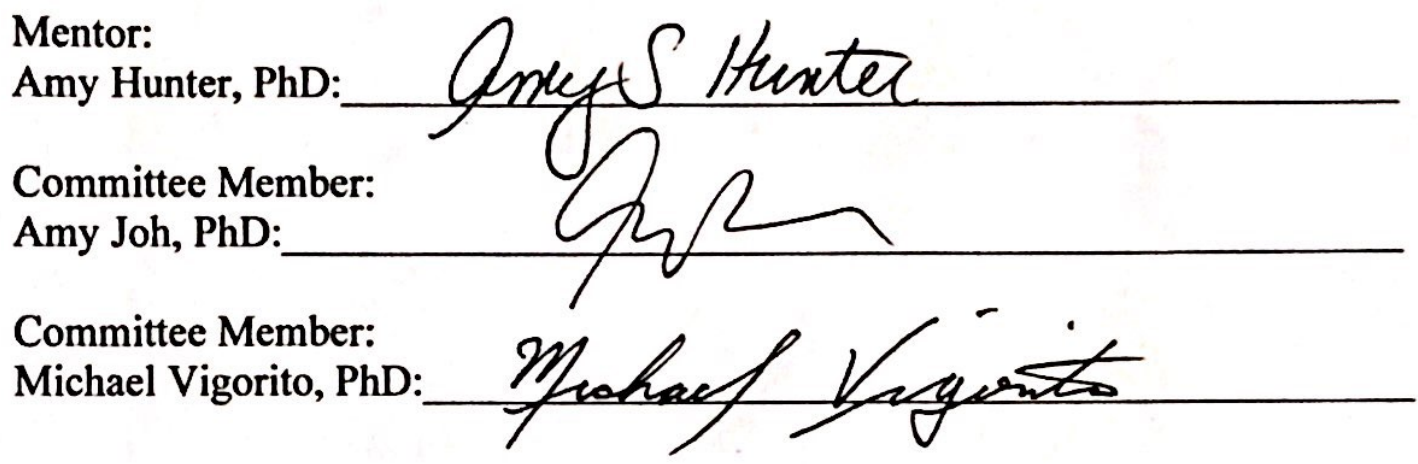




\section{Acknowledgements}

I would like to thank my thesis advisor, Dr. Amy Hunter, as well as my committee members, Dr. Amy Joh and Dr. Michael Vigorito. Thank you for giving your time, insight, and encouragement throughout this process. I have learned more from you than we can quantify. I would also like to thank Kaitlyn Heywood, Kelly Mannion, Nadia Meshkati, and Naquan Ross. From aiding in coding data to walking my dog when I could not, this project would not have been possible without you.

Finally, I want to give a special thank you to my parents, Stephen, and Jonny. To my parents, who make opportunities like this possible and give endless love and support. To Stephen, who is unwavering in his positivity and encouragement. And to Jonny, who keeps me company, keeps me sane, and barks to tell me when it is time to put down the computer and go to bed. 


\section{Table of Contents}

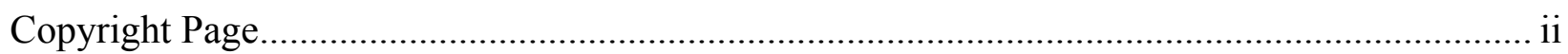

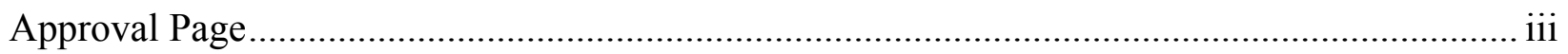

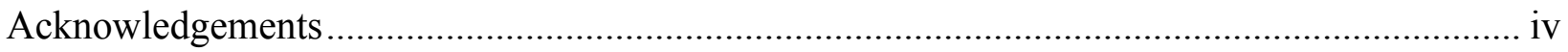

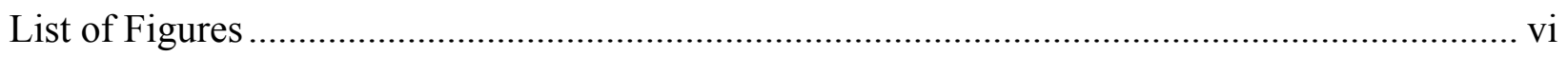

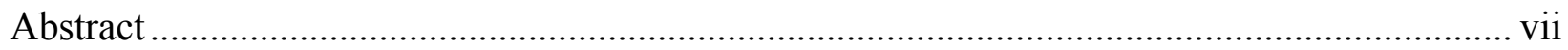

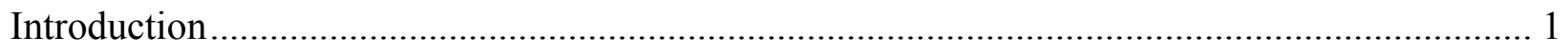

Experimental Sleep Deprivation with Rodent Models ...................................................... 5

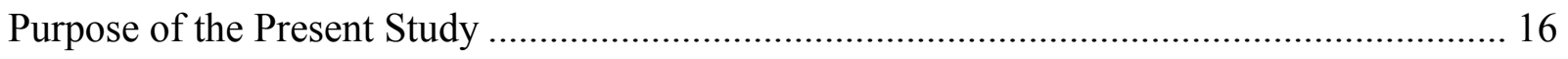

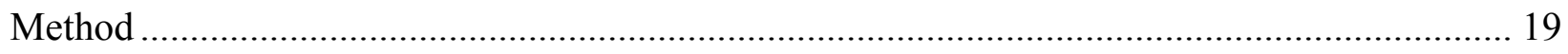

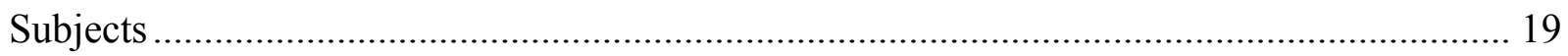

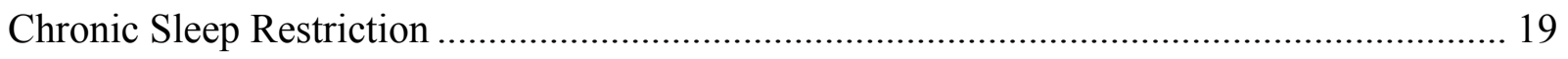

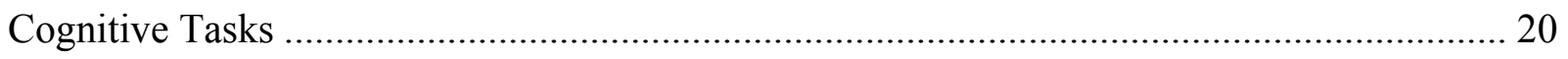

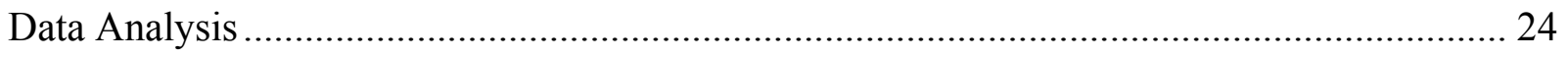

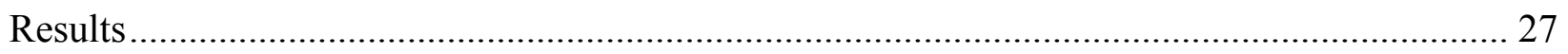

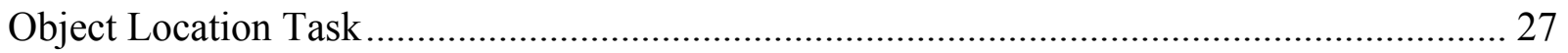

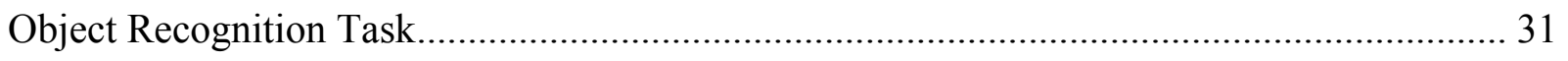

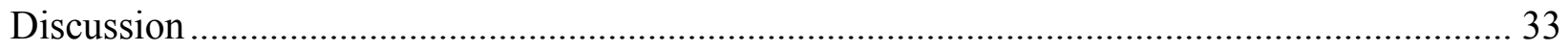

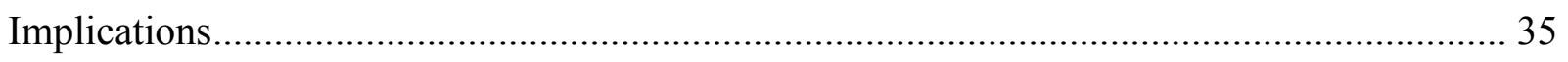

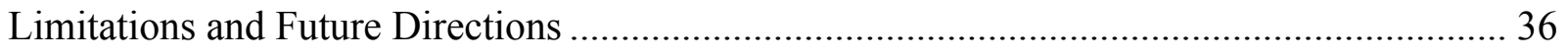

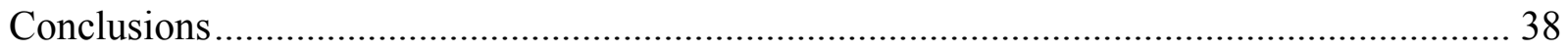

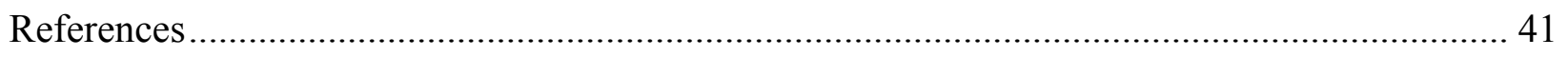

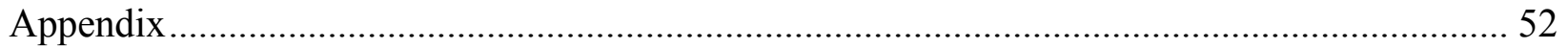




\section{List of Figures}

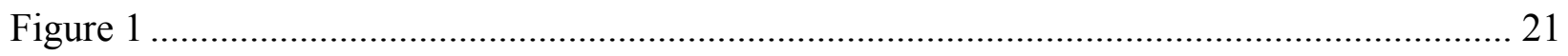

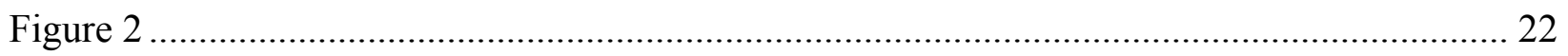

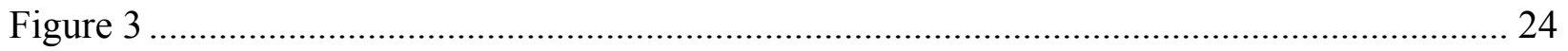

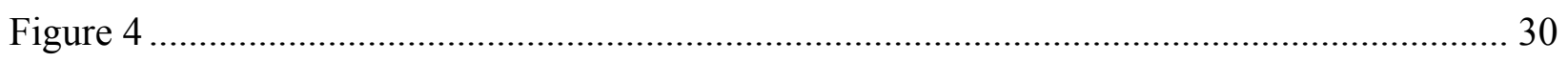

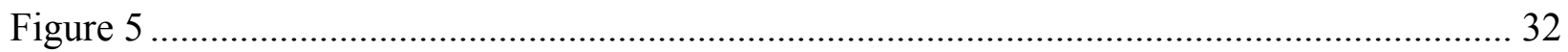




\begin{abstract}
Adolescence is a critical developmental period. An important change that occurs in adolescence is the neurological maturation for adult-type cognitive abilities. Research has linked adequate sleep quantity to successful learning and memory capabilities. However, due to a shift in sleep timing drive in adolescence, in combination with early awakening for school, the adolescent population is experiencing chronic sleep restriction (CSR). What repercussions to long-term memory capabilities could CSR in adolescence have immediately and are the consequences longlasting? The present study modeled human adolescent CSR in rats through four hours of sleep deprivation for five days, followed by two days of unrestricted sleep, and five more days of four hours of sleep deprivation; thus the rats were exposed to CSR throughout the two-week rat adolescent period. Long-term hippocampal dependent and non-hippocampal dependent memory were tested through the object location task and the object recognition task, respectively. Testing occurred in adolescence and after a four-week delay during which the rats slept freely and matured to adulthood. The results showed that, given the appropriate conditions for successful long-term memory, the rats exposed to CSR in adolescence showed impaired hippocampal dependent memory in adolescence and this impairment was also evident in adulthood. These findings were not the case for non-hippocampal dependent memory, for which a significant effect of sleep was not found. Given the findings of the hippocampal dependent task, these results suggest that CSR in adolescence may influence less than optimal memory performance among adolescents. Further, the pattern in adulthood suggests that even after undisturbed sleep in the period from adolescence to adult maturation, the consequences of adolescent CSR are relentless. The findings in this study inform the research as the first rodent model of adolescent CSR and indicate practical implications for the health of adolescents.
\end{abstract}




\section{Introduction}

Humans spend a third of their lives sleeping. Based on recommendations from the National Sleep Foundation, a person who lives an average life expectancy and gets the appropriate amount of sleep for his or her age across the lifespan will average approximately 8.6 hours of sleep per night — over a third of our 24-hour days (Global Burden of Disease 2015 Mortality and Cause of Death Collaborators, 2016; Hirshkowitz et al., 2015). The considerable time that humans spend sleeping implies that it must serve an imperative function. Research asserts that sleep is primarily for the brain with a demonstrated necessity in optimal cognitive functioning and neurological maturation (Frank, 2006; McCoy \& Strecker, 2011; Poe, 2017; Sara, 2017). One population to which this function is of particular concern is adolescents, who are in a critical developmental period for the establishment of ideal adult-type neurology and cognition (Lenroot \& Giedd, 2006; Sisk \& Zehr, 2005). Therefore, optimal sleep quantity is vital in the adolescent period. Unfortunately, optimal sleep quantity is precisely the opposite of the pattern seen among today's adolescents.

Despite evidence of the importance of sleep, too few people are getting the recommended amount (Bonnet \& Arand, 1995; Schoenborn \& Adams, 2010) with the adolescent population falling most drastically into this category (Carskadon, 2011). Naturally, adolescent sleep schedules are constrained by a biological shift from morning to evening chronotype (staying up later due to the combination of changes in circadian timing and a delay in homeostatic build-up) and morning social demands, such as school (Hagenauer \& Lee, 2013). Further, additional factors such as late night phone use and caffeine consumption may intensify the natural delay (Crowley et al., 2014; Owens, 2014). The repetitive nature of the insufficient sleep (i.e., limited sleep for multiple days in a row on weekdays) indicates that adolescents are experiencing chronic 
sleep restriction (CSR; e.g., Beebe, Rose, \& Amin, 2010; Carskadon, 2011; Jiang et al., 2011). In fact, while about $65 \%$ of adults are getting their recommended $7-8$ hours of sleep, only $20 \%$ of teens are getting the optimal 8-10 hours of sleep on school nights, attesting to the issue's prevalence in today's society (Hirshkowitz et al., 2015; Lui et al., 2016; National Sleep Foundation, 2006; Owens, Belon, \& Moss, 2010).

In addition to the demonstrated health-related consequences of insufficient sleep quantity (e.g., increased risk for obesity, type 2 diabetes, and cardiovascular complications; Nagai, Hoshide, \& Kario, 2010; Spiegel, Knutson, Leproult, Tasail, \& Van Cauter, 2005), research asserts that sleep is primarily for the brain, implicating it in cognitive function and mental health (Frank, 2006; Owens, 2014). In particular, there is substantial evidence of sleep's role in learning and memory (for reviews see Poe, 2017 and Sara, 2017). Studies in which learning is either preceded or followed by a period of sleep deprivation consistently show impaired acquisition of the task and memory for the task, respectively (see McCoy \& Strecker, 2011 for review). The pattern of impairment caused by sleep deprivation implicates sleep in learning and memory, with cognitive deficits independent of the decreased alertness that inevitably accompanies extended wakefulness (Smith \& Smith, 2003).

The present understanding is that sleep is important to the memory consolidation process; that is, sleep secures memories through transferring them to long-term memory and making them less susceptible to interference (McCoy \& Strecker, 2011). Furthermore, sleep seems to be involved in forgetting as well. During sleep, information undergoes a targeted eliminating process involving the weakening of specific synaptic connections, so as to not crowd the brain and its synapses with irrelevant, incorrect, or conflicting information (Poe, 2017). Consequently, it appears that sleep is important across the learning and memory spectrum, from initial 
acquisition and encoding to recollection and long-term stabilization to freeing of synapses so that new learning may occur.

The importance of sleep to learning and memory processes, in collaboration with how it changes across the lifespan, hints towards developmental implications. The recommended quantity of sleep for optimal functioning varies across the lifespan: an average of 14-17 hours per day are recommended for newborns, 10-13 hours for preschoolers, 8-10 hours for adolescents, and 7-8 hours for adults, to give some examples (Hirshkowitz et al., 2015). The differential hours based on ages asserts more sleep necessity during development than at maturation. Additionally, minutes spent in the stages of sleep that are important to learning and memory are greatest throughout childhood and adolescence and do not stabilize to adult levels until early adulthood, corresponding to the brain maturation that occurs through childhood and adolescence and into early adulthood (Carskadon \& Dement, 2011; Capellini et al., 2008; Spear, 2013; Stickgold, James, \& Hobson, 2000; Ohayon, Carskadon, Guilleminault, \& Vitiello, 2004). Once the brain is more fully developed in adulthood, both recommended hours of sleep and time spent in these crucial stages lessen. Given the elevated recommended number of hours of sleep and time spent in stages that are concurrent with learning and memory processes, it follows that sleep corresponds to cortical growth and connectivity associated with neurological development.

Adolescence is a particularly critical developmental period for the establishment of ideal adult-type neurology and cognition. Adolescence is a period of physical, hormonal, and neurological changes as the body makes the transition from childhood to adulthood (Sisk \& Zehr, 2005). While some neurological maturation has reached peak growth or volume in childhood (e.g., total cerebral volume reaches $95 \%$ of its peak volume around age six and the amount of gray matter for the frontal and parietal lobes peaks around 10-12 years), the crucial 
refinement — reduction, in some cases — of such areas and preparation of adult-type patterns occurs in adolescence (Lenroot \& Giedd, 2006; Sisk \& Zehr, 2005). Essential changes in adolescence include specialized pruning to refine connections, the establishment of more efficient synapses, and an increase in the production of myelin for speedier processing (Spear, 2013). The increase in the production of myelin corresponds to an increase in white matter in areas specialized for adult cognition (Bava \& Tapert, 2010; Lenroot \& Giedd, 2006). Furthermore, areas that mature later in the process, such as frontal regions and the prefrontal cortex, continue to develop during adolescence (Spear, 2013). As such areas have been implicated in inhibitory control and the ability to manage emotional situations, their continued development in adolescence accounts for the impulsivity that is seen in this period, while also indicative of progression toward adult-type patterns and eventual behavior.

With sleep's importance in neurological maturation and these crucial preparations for an adult brain system that occur in adolescence, optimal sleep quantity is vital in the adolescent period. However, later sleep onset in adolescence and continued early awakening leaves fewer hours for sleep. That is, morning social demands may be hindering a natural disposition to sleep later into the day (Crowley et al., 2014). For example, a child in early middle school may go to bed at 9:00-10:00pm and wake up at 6:00-7:00am, generating an average of about 8-9 hours of sleep; an adolescent in high school may not go to bed until 11:00pm-midnight, while still having to wake up at 6:00-7:00am, generating an average of only about 6-7 hours of sleep (National Sleep Foundation, 2006). These 6-7 hours are in contrast to the 8-10 hours that are recommended for this crucial developmental stage (Hirshkowitz et al., 2015). What cognitive repercussions is society generating on the dynamic and vulnerable adolescent brain, both immediately and in a long-term sense, with this CSR? 


\section{Experimental Sleep Deprivation with Rodent Models}

A substantial amount of literature has examined the immediate consequences of CSR in humans, such as impaired sustained attention, daytime sleepiness, and lower quiz scores (e.g., Agostini, Carskadon, Dorrian, Coussens, \& Short, 2016; Beebe et al., 2010). Additionally, studies have shown that insufficient sleep during development can predict long-lasting difficulties, such as increased incidence of mental illness and addiction (see Beebe, 2011 for review). However, the nature of experimentation makes such questions difficult to answer with human subjects. That is, a researcher may lack full control of the sleep manipulation or the ability to use a comparative control group with random assignment to groups, thus opening the study to a lack of internal validity. Thus, while both immediate and longitudinal studies with humans exist, they are unable to make causal statements and tend to focus on resulting mood disorders and substance abuse, rather than on strictly cognitive abilities.

With such limitations in mind, sleep researchers have pushed for the use of rodent models to study the cognitive consequences of sleep disturbance (McCoy \& Strecker, 2011). In addition to appeasing these limitations, rat models boast the benefit of providing insight into neurological mechanisms and investigating long-lasting effects is more feasible, as the change from adolescence to adulthood in rodents occurs over the course of weeks, as opposed to years. Given such benefits, laboratory manipulations with rats have been developed to model sleep disorders. For instance, sleep fragmentation (the periodic awakening of the rat) has been used to model sleep apnea (Ward et al., 2009). On the other hand, while researchers have modeled CSR with rats to examine the sleep architecture and neurobiological changes that result from CSR (e.g., Kim, Laposky, Bergmann, \& Turek, 2007; Leemburg et al., 2010), its application as a potential 
model of adolescent sleep schedules and the cognitive consequences of such constraint has received little attention.

Sleep research with rodents comes with it the obvious problem of generalizing findings from a non-human species to humans. Some researchers argue that results with rodents cannot be generalized in this manner (e.g., Shanks, Greek, \& Greek, 2009). However, in this case of investigating changes in adolescence, Spear (2000) reviews a number of similarities between rodents and humans that make the case for rodents as an acceptable human model. For example, adolescent rats show the phase delay in the onset of sleep that is evident in human adolescents, suggesting that both species have the natural tendency to stay up later that is contributing to the CSR experienced by human adolescents. Additionally, neurological alterations evident in adolescent humans, such as myelination, synaptic pruning, and maturation of the prefrontal cortex that contribute to cognitive and behavioral changes, are also seen in rats (Bellesi et al., 2018; Spear, 2000; Spear, 2013; Brenhouse \& Andersen, 2011). While Spear (2000) admits that animals cannot perfectly model humans, adolescent developmental changes, in particular, seem remarkably similar across species (see Brenhouse \& Anderson, 2011 for review).

Learning and memory. The impairment in cognitive abilities as a result of sleep disturbances in rodents has been extensively examined among researchers. Learning and memory have received the most consideration. The consideration is likely due to the wide variety of forms of memory (e.g., working memory, reference memory, motor memory, recognition memory, spatial memory, long-term memory, etc.) and the intrigue generated by how these systems are impaired in diverse ways by employment of alternative tasks, inducing different types of sleep impairments, or altering the timing of sleep impairment. For example, total sleep deprivation and sleep fragmentation (periodic awakening throughout a sleep phase) following 
training of a maze task may impair spatial memory in an adult rat; however, these impairments may be in contrast to those produced by deprivation of only the rapid eye movement (REM) stage of sleep which may leave spatial memory intact, while debilitating reference memory and emotional memories (e.g., Guan, Peng, \& Fang, 2004; Smith, Conway, \& Rose, 1998; Walsh, Booth, Poe, 2011; Walker, 2010; Ward et al., 2009).

The forms of information that are learned and stored in memory can be broadly classified as hippocampal dependent and non-hippocampal dependent. The hippocampus is known for its role in tasks that require spatial or contextual skill (D'Hooge \& De Deyn, 2001; O'Brien, Lehmann, Lecluse, \& Mumby, 2006). To give an example of this distinction, the role in spatial skills is demonstrated through lesion-induced impairment in performance on spatial tasks, such as the popular Morris water maze (MWM; Morris, 1984), in which animals are required to learn the location of a hidden platform. The rat can be placed into the apparatus at various start locations, thus requiring the hippocampus for memory of the location of the platform and use of external cues to navigate towards it. By contrast, the MWM can also be set up to not require the hippocampus, by placing the rat into the apparatus at the same location each time and requiring a learned motor pattern, rather than spatial memory (Carlson, 2013). Further, a number of neuroimaging studies implicate a variety of non-hippocampal brain regions in different types of memory. For example, working memory maintenance has been linked to frontoparietal activity and episodic memory encoding to prefrontal structures, thereby illustrating memory that is not primarily linked to the hippocampus (Eriksson, Vogel, Lansner, Bergström, \& Nyberg, 2015; Tulving \& Markowitsch, 1997). With the hippocampal/non-hippocampal dependent classification in mind, researchers may choose and alter experimental tasks based the form of learning and memory of interest. 
The object location task and object recognition task. The object location task (OLT; Dix \& Aggleton, 1999) and object recognition task (ORT; Ennaceur \& Delacour, 1988) tap into distinct types of learning and memory. The OLT is a spatial task requiring the subject to differentiate between two object locations, while the ORT is a recognition task requiring the subject to differentiate between a novel object and a familiar object. The OLT's reliance on the hippocampus is indicated in a study by Ennaceur, Neave, and Aggleton (1997) in which lesions to pathways to the hippocampus impaired task performance. On the other hand, the ORT is a recognition task that appears reliant on alternative areas, such as the perirhinal cortex, the insular cortex, and the ventromedial prefrontal cortex (Akirav \& Maroun, 2006; Balderas et al., 2008; Ennaceur et al., 1997; O’Brien, Lehmann, Lecluse, \& Membuy, 2006; Winters, Forwood, Cowell, Saksida, \& Bussey, 2004). The OLT and ORT demonstrate the distinction between hippocampal dependent and non-hippocampal dependent memory.

Despite tapping into different mechanisms, the OLT and ORT utilize similar methodology that is reliant on rodents' instinctual novelty preference (Dix \& Aggleton, 1999). In the OLT, one object is in the same spot as in a previous exposure to the objects and one object has been moved to a new location within the apparatus. By comparison, in the ORT, the two objects remain in the same location, but one of the objects is different from the object in the previous exposure (Ennaceur \& Delacour, 1988). The subjects' learning and memory from the first exposure (the study phase) to the test phase is quantified based on rodents' innate behavior to display more attention towards a novel object or an object in a novel position. That is, successful spatial and recognition learning and memory in these two tasks manifests in preference for the object in the novel location and the novel object, respectively (Strecker, Drinkenburg, Sahgal, \& Aggleton, 1998). 
As rodents innately show exploratory preference for novelty over familiarity, the OLT and ORT do not require lengthy shaping or training procedures (Ennaceur \& Delacour, 1988). The lack of training provides an advantage over a number of other tests of cognitive abilities, such as the 6-day pre-training phase of the delayed non-matching-to-sample task (Mumby, Pinel, \& Wood, 1990) or the 3-5 month training period of the 5-choice serial reaction time task (Cordova et al., 2006). The OLT and ORT can be accomplished in a relatively short timeframe and offer a snapshot of memory abilities at a precise time point during development. Additionally, they require no external motivation or potentially stress-inducing procedures (Ennaceur \& Delacour, 1988; Silvers, Harrod, Mactutus, \& Booze, 2007). The lack of these potentially confounding variables boasts comparability to learning and memory studies with humans who can be told or read instructions for a task without training or motivation through food or water deprivation and rewards. Therefore, the OLT and ORT allow the researcher to investigate hippocampal dependent memory and an example of non-hippocampal dependent memory, recognition memory, through instinctual rat behaviors.

The necessity to investigate both hippocampal dependent memory and non-hippocampal memory is evident in the sleep literature, as sleep deprivation may differentially impair tasks based on the tasks' reliance on the hippocampus. Palchykova, Crestani, Meerlo, and Tobler (2006) deprived hamsters of four hours of sleep, through gentle handling, after ORT training. Palchykova, Winsky-Sommerer, Meerlo, Dürr, and Tobler (2006) extended this study to mice, depriving them of six hours of sleep after learning the ORT, as four hours for a hamster is comparable to six hours for a mouse (Palchykova, Crestani et al., 2006). In both studies, sleepdeprived animals failed to exhibit a distinction between novel and familiar objects at test, 24 hours later, in the non-hippocampal dependent ORT. In addition, sleep-deprived hamsters failed 
to differentiate between the object in the familiar location and the object in the novel location in the hippocampal-dependent OLT (Palchykova, Crestani et al., 2006). This impairment provides evidence that a single instance of sleep deprivation impairs both spatial and non-spatial memory in rodents. However, in Palchykova, Winsky-Sommerer, and colleagues (2006), even the control mice failed to distinguish between an object at a novel location and an object at its prior location. Therefore, in this case, the OLT was not sensitive enough to pick up differentiation between novelty and familiarity, unrelated to any sleep manipulation. While the ORT was sensitive to sleep deprivation in both studies, the results of Palchykova, Winsky-Sommerer, and colleagues with mice leave questions regarding the effect of sleep deprivation on the OLT.

The inconclusiveness of the studies by Palchykova and colleagues for the OLT is in contrast to a study by Binder and colleagues (2012). Binder and colleagues found that successful differentiation between novel location and familiar location was demonstrated only in the morning (inactive phase, when rats will presumably sleep) timeframe without sleep deprivation. This finding implies that sleep following acquisition is important to hippocampal dependent learning and memory and, therefore, future performance on the task. These findings provide evidence that sleep is essential to performance on the OLT, as well.

Ngo, Lloyd, and Hunter (2013) used an alternative form of sleep deprivation, selectively depriving rats of REM for six hours. The results showed that REM-deprived rats had less preference for novel objects (ORT impairment) than control rats. On the other hand, the results for the OLT were less straightforward because the within-subjects design resulted in evidence of a carryover effect. That is, while rats that first learned the OLT and were subsequently deprived of REM did not show an effect of REM deprivation on the OLT, rats that were REM deprived first for the ORT, prior to the acquisition of the OLT did show impairment. Thus, these findings 
contribute the consequences of REM deprivation to the literature on impairment of the ORT, while, as was the case with the mice in the study by Palchykova, Winsky-Sommerer, and colleagues, sleep disruption's effect on the OLT was inconclusive.

Chronic sleep restriction. While the OLT and ORT have not yet been used to study the effects of CSR, Hairston et al. (2005) and McCoy et al. (2013) have investigated cognitive impairment in response to CSR with the MWM. McCoy and colleagues applied a severe CSR sleep manipulation of 18 hours per day for five days (only 6 hours of sleep opportunity per day). The results showed that CSR rats demonstrated impairment in memory for platform location. Hairston and colleagues induced a comparatively mild sleep manipulation of 6 hours per day for four days. The results were similar to those of McCoy and colleagues, in that the CSR group took longer to find the platform than undisturbed rats, suggesting hippocampal dependent memory impairment. In a non-hippocampal dependent version of the task, the platform was raised above the water such that it was visible. Therefore, swimming to the platform did not require the use of spatial cues. In contrast to the results of the hippocampal dependent test, the CSR rats showed improvement in this version of the task. The authors concluded that the positive performance on this task for the CSR group is an indication of CSR altering the type of memory strategy employed by the rats. As a conclusion, there is evidence that CSR produces spatial memory deficits in adult rats and lack of evidence that CSR shows that same effect in non-spatial tasks.

Summary. Given the studies discussed thus far, we see that researchers have explored the effects of sleep disturbances of varying types on cognitive abilities in rat models. However, CSR has less often been the deprivation type of choice in experimental contexts than alternative sleep deprivation methods (McCoy et al., 2013) and it has yet to be analyzed with the OLT and ORT. 
Therefore, while there is substantial literature on cognitive impairments from sleep disturbance, there are still questions about what skills are affected by CSR, specifically. For example, varied findings are demonstrated in impairment to hippocampal dependent memory as a result of CSR in the MWM, but a lack of impairment to non-hippocampal dependent memory (Hairston et al., 2005; McCoy et al., 2013). Investigating the effects of CSR on the OLT and ORT may provide useful information about whether CSR in adolescence affects both hippocampal dependent and non-hippocampal dependent learning and memory.

Adolescence. A significant question among adolescent studies with laboratory animals is what ages in rodents corresponds to human adolescence? Vetter-O'Hagen and Spear (2012) measured physiological and hormonal markers of puberty in Sprague-Dawley rats (e.g., genital development, weight gain, hormone levels) and surmised a wide timeframe for adolescence of post-natal days (PND) 25-55. The proposed timeframe can be divided into comparative subgroups: PNDs 25-27 correspond to ages 10-12 in humans, PNDs 28-42 correspond to ages 12-18 in humans, and PNDs 43-65 correspond to 18-25 years of age (late adolescence and emerging adulthood) (Spear, 2000; Spear, 2015; Vetter-O’Hagen \& Spear, 2012). While these definitions may differ from the period put forward by other rat adolescent studies, it is also important to note that differences may exist between various strains of rats and, for the present study, literature that used Sprague-Dawley rats was prioritized when determining logistical factors of the experiment (Harker \& Whishaw, 2002; McCoy et al., 2013).

Regarding the timing of adulthood, the above studies for operationally defining adolescence in rats signified PND 65 as the end of the emerging adulthood period, suggesting that adulthood would subsequently begin. The proposed timing corresponds to additional studies that have examined the long-lasting effects of an adolescent manipulation on characteristics of 
adulthood. For example, recently Saré, Levine, Hildreith, Picchioni, and Smith (2016) and Billeh and colleagues (2016) identified approximately 4-5 weeks post-adolescent manipulation as their adulthood period (31 days later at PND 73 and 35 days at PND 65, respectively). While Saré and colleagues and Billeh and colleagues used mice for their experiments, a study with SpragueDawley rats (Yang, Sun, Huang, Yao, \& Qu, 2012) utilized a similar timeframe, operationally defining adulthood as within PND 60-85. Again, these studies are in agreement with the aforementioned studies regarding late adolescence and emerging adulthood (PND 43-65); thus, the literature suggests that after PND 65 is an agreeable estimate to ensure that, on average, rats can be considered adults.

Chronic sleep restriction. While the cognitive repercussions of sleep disturbance have been investigated, those studies were performed with adult rats and few studies have looked to answer questions regarding impairments in adolescent rats. In fact, to our knowledge, only one study has done so. Yang and colleagues (2012) deprived both adolescent and adult rats of four hours of sleep for seven days, using gentle handling, to investigate the effects on hippocampaldependent memory using the MWM. The adolescent manipulation was implemented for PND 29-34 and a separate group of rats underwent the adult manipulation at PND 72-79. Results showed that CSR impaired the performance of adolescent rats who, compared to undisturbed controls, travelled further to find the platform, spent less time in the platform quadrant, and made fewer platform crossings. This finding is in opposition to the adult rats for which the authors did not find impairment in any of these measures. The lack of impairment in adults is in contrast to studies demonstrating in impairment in the MWM as a consequence of CSR in adult rats (Hairston et al., 2005; McCoy et al., 2013). However, studies that found deficits using the MWM employed longer periods of CSR, suggesting that an extended period of CSR may be necessary 
to produce MWM impairment. While cross-sectional in nature, this study of Yang and colleagues does provide preliminary evidence for impairment to cognition (namely, spatial memory), revealing the potential for immediate deficits from CSR in adolescence, but did not investigate possible long-lasting effects.

Immediate and long-lasting effects. Despite the lack of research into cognitive deficits with adolescent rodents, research with laboratory animals has delved into the vulnerability of the adolescent brain to issues other than cognitive impairments after sleep deprivation. CSR studies, specifically, have noted both neurological and behavioral changes or deficits and, in some cases, have demonstrated changes in adulthood stemming from CSR manipulation in adolescence.

Saré and colleagues (2016) linked CSR during development with behavioral abnormalities. Saré and colleagues deprived mice of three hours of sleep per day through gentle handling. The sleep manipulation was employed for 38 days, from childhood through adolescence (PND 5-42). Differences between the sleep deprived mice and a group of controls were examined on a number of factors: sociability, anxiety, motor function, and repetitive behaviors (marble burying). The CSR group showed less exploratory behavior two days after the completion of deprivation procedures, indicated by less distance traveled in the open field; an increase in sociability one day after sleep deprivation, indicated by a task that looked at preference for a stranger mouse over an object; and a decrease in repetitive behaviors immediately following deprivation, indicated by less marble burying. These findings indicate the immediate effects of CSR on these behavioral measures.

Long-lasting effects of CSR were also exhibited, as Saré and colleagues also examined these factors at PND 73 after four weeks of sleep recovery. As was observed two days after deprivation, the CSR group showed less exploratory behavior in the open field. Sociability and 
repetitive behaviors, on the other hand, showed sleep and sex differences that were not apparent earlier. In the sociability task, sleep-deprived male rats preferred to sniff an object over social novelty in the form of a stranger mouse. However, sleep-deprived females retained an increased preference for the stranger mouse. In a second measure of sociability, preference for a novel stranger mouse, an effect of the sleep manipulation was not observed. Finally, sleep-deprived females showed less repetitive behaviors than controls. Regardless of differences in the exact pattern of change, this study provides evidence of long-lasting behavioral change as a result of CSR during development.

Additionally, a study by Billeh et al. (2016) implemented a CSR manipulation in early adolescence (PND 25-29) with mice and investigated long-lasting effects by looking at abnormalities in brain connectivity in adulthood (imaged between PND 65 and 68). The experimental group was deprived of $50-60 \%$ of sleep for five days through exposure to novel objects, environments, or social interaction, in combination with forced locomotion. The objective was to view projections from the secondary motor cortex, but they were only able to find "subtle and heterogeneous" variation as an effect of adolescent CSR on adult brain connectivity (Billeh et al., 2016, p.13). That is, differences tended towards less projections in the CSR group, but the degree of the effect resulted in a non-significant difference overall. The authors brought up such concerns, noting that the study is elementary — the first among the research to examine if consequences of CSR in adolescence prevail in the adult brain of laboratory animals.

On the other hand, Novati, Hulshof, Koolhaas, Lucassen, and Meerlo (2011) did show immediate neurological effects of CSR in adolescent rats. Their study implemented one of the greater amounts of CSR at approximately 20 hours a day for a month (PND 30-61), achieved 
through rotating drums. The results demonstrated $10 \%$ decrease in dorsal hippocampal volume in the CSR group. This finding was consistent with data on human sleep disorders, such as insomnia and sleep apnea, both of which are characterized by a reduced volume of the hippocampus (Morrell et al., 2003; Riemann et al., 2007). Evidence for the reduced volume has also been seen in mood disorders (e.g., Czéh \& Lucassen, 2007; Sapolsky, 2000). Taken together, this line of research demonstrates the importance of sufficient sleep in adolescence and suggests that the lack of it may be involved in abnormalities that can be associated with additional sleep problems and psychological disorders.

Summary. Based on the body of adolescent cognition research with rodents, Yang and colleagues (2012) have, to our knowledge, conducted the sole investigation into the cognitive consequences of CSR in adolescence. Their study provides the present study with foundational evidence of memory deficits as a result of adolescent CSR. Their findings speak to the vulnerability of the adolescent brain, as do a number of other studies in which sleep disturbances in adolescence show immediate and, in some cases, long-lasting changes (Billeh et al., 2016; Novati et al., 2011; Saré et al., 2016; Ribeiro-Silva et al., 2016). The demonstrated vulnerability of the adolescent brain to sleep deficiency and the gap in research on resulting cognitive impairments reinforces the need for research on the potential immediate and long-lasting cognitive deficits that may result from the CSR that is common in adolescence.

\section{Purpose of the Present Study}

Previous research with laboratory animals has investigated the learning and memory deficits caused by a variety of sleep deficiencies, as well as the consequences of sleep deficiency in adolescence. However, the research has yet to sufficiently explore how these topics overlap and doing so is the objective of the current study. Yang and colleagues (2012) is the closest of 
the literature to this objective, but while their study was interested in age-related differences in resistance to the effects of CSR, the present study is interested in the effects of a sleep manipulation that is administered in adolescence on present and long-lasting cognitive abilities. Therefore, the present study attempted to model, in rats, the CSR seen in human adolescents in order to investigate ensuing cognitive impairments in the immediate sense and whether they carry into adulthood. We implemented CSR to adolescent rats (PND 28-42) in a similar schedule: five days of CSR, followed by two days of undisturbed sleep, followed by another five days of CSR. This schedule mimicked human adolescent sleep deprivation by delaying sleep onset on week nights, allowing for the potential rebound of some sleep quantity during an undisturbed two-day "weekend," and then repeating the restriction during the week (Beebe et al., 2010; Hagenauer \& Lee, 2013; Owens et al., 2010). Based on previous research, we predicted that CSR would impair performance on both the hippocampal dependent OLT and the nonhippocampal dependent ORT when tested in adolescence.

Given the fact that adolescence is a critical developmental period, the present study also investigated possible long-lasting consequences of CSR. We hoped that the increase in CSR days to 10 from the 7 applied by Yang and colleagues (2012) would improve chances of revealing long-term effects in adulthood. The same rats also underwent testing four weeks later at PND 67 and 70 in an attempt to investigate not only immediate effects of CSR in adolescence, but also if adolescent CSR effects on cognition persist into adulthood. The four-week delay is a dependable timeframe for examining the long-lasting effects to adulthood of an adolescent manipulation as PND 65 coincides with adulthood in the rat (Saré et al., 2016; Billeh et al., 2016; Spear, 2015). We predicted that there would be consistent pattern of impairment in adolescence and adulthood. 
By using the OLT and ORT in conjunction with CSR, the present research fills a hole in the literature, as the cognitive impairment of CSR has not been examined with these tasks. The specific cognitive impairments of interest were hippocampal dependent (OLT) and nonhippocampal dependent (ORT) long-term memory. In order to check that we were indeed tapping into long-term memory mechanisms, we used two different study phase timings. Prior research with the OLT shows that a 20 min study phase is necessary to produce any abovechance discrimination between the novel and familiar object locations at test 24 hours later, while a 5 min study phase is too short to result in memory for the object (Ozawa, Yamada, \& Ichitani, 2011). As such, we expected neither rats exposed to CSR nor control rats to show successful long-term memory in the 5 min study phase, but that the CSR rats would show impaired long-term memory in the 20 min study phase, compared to successful memory in the control rats. To summarize, our hypotheses anticipated a study phase x sleep condition interaction, with the lack of main effect for age in this interaction indicative of a similar pattern of impairment in adolescence and adulthood, signifying long-lasting consequences. 


\section{Method}

\section{Subjects}

Subjects were 32 adolescent male Sprague-Dawley rats. Adolescence was operationally defined as PND 28-42, approximately ages 12-18 years in humans (Spear, 2000; Spear, 2015; Vetter-O’Hagen \& Spear, 2012). Animals were obtained from Envigo at PND 21 and given a week to habituate to the lab. Animals were housed with two rats per cage (except during sleep manipulation procedures) and under constant temperatures $\left(23^{\circ} \mathrm{C}\right)$. The light/dark cycle was 12:12 (lights on at 08:00am). Food (Envigo lab chow) and water was available to the rats ad libitum throughout the experiment. The experiment obtained IACUC approval prior to its start and followed the guidelines of the United States Public Health Service. A power analysis with medium effect size and $\alpha=.05$ showed that 32 total animals were required for power above $80 \%$ (82.23).

\section{Chronic Sleep Restriction}

Sleep deprivation was achieved through mild stimulation and gentle handling (e.g., tapping or shaking the cage to keep the animal awake and petting or picking up the animal if the stimulation ceased to be effective; Hagewoud et al., 2010; Yang et al., 2012). Mild stimulation and gentle handling procedures were implemented upon observation of the rat curling up with its eyes closed. Half of the subjects underwent the sleep manipulation $(n=16)$, while the other half constituted an undisturbed control group and was allowed to sleep ad libitum in their home cages in the colony room for the duration of the study. CSR was implemented from 8:00am-12:00pm (four hours). During deprivation procedures, rats were housed individually. Upon completion of the four hours of CSR, rats were returned to home cages. We housed a CSR rat and a control rat together for the duration of the study. 
The sleep manipulation continued over two weeks, with a two-day "weekend" of undisturbed sleep included. Thus, adolescent rats experienced CSR of four hours/day for five days, PND 28-32; undisturbed sleep for two days, PND 33-34; and CSR of four hours/day for five days, PND 35-39. The complete protocol is shown in Figure 1.

\section{Cognitive Tasks}

Sixteen rats ( 8 from the CSR group and 8 from the control group) completed the OLT and 16 rats ( 8 from the CSR group and 8 from the control group) completed the ORT. A between-subjects design in which subjects only completed one of the two cognition tasks was chosen to eliminate potential task order effects (Ngo et al., 2013). All stages of the tasks occurred prior to the sleep phase (7:00am-8:00am).

Apparatus and objects. The apparatus for the tasks was four identical 67.8 x $40.1 \times 35.3$ cm semi-opaque plastic bins (similar to Ennaceur and Delacour, 1988; Ngo et al., 2013). The two bins for the OLT were marked with black and white stripes and a target on the front and back

ends of the apparatus to provide spatial cues (see Figure 2). The two bins for the ORT were not marked in any way. 


\begin{tabular}{|c|c|c|c|c|c|c|c|c|c|c|c|c|c|c|c|c|c|c|c|c|c|c|c|}
\hline PND & 28-31 & & 32 & & & & & & 35 & & & & & 38 & & & 40 & $40-66$ & 66 & 67 & 68 & 69 & 70 \\
\hline $\begin{array}{l}\text { CSR-L } \\
\text { Group }\end{array}$ & CSR & $\mathbf{H}$ & CSR & $\mathbf{H}$ & $\mathbf{U}$ & H & $\mathbf{U}$ & $\mathbf{H}$ & CSR & $\begin{array}{c}\text { S1 } \\
\text { OLT }\end{array}$ & CSR & $\begin{array}{c}\text { T1 } \\
\text { OLT } \\
\end{array}$ & CSR & CSR & $\begin{array}{c}\text { S2 } \\
\text { OLT }\end{array}$ & CSR & $\begin{array}{c}\text { T2 } \\
\text { OLT }\end{array}$ & $\mathbf{U}$ & $\begin{array}{c}\text { S3 } \\
\text { OLT }\end{array}$ & $\begin{array}{c}\text { T3 } \\
\text { OLT }\end{array}$ & $\mathbf{U}$ & $\begin{array}{c}\text { S4 } \\
\text { OLT }\end{array}$ & $\begin{array}{c}\text { T4 } \\
\text { OLT }\end{array}$ \\
\hline $\begin{array}{l}\text { CSR-R } \\
\text { Group }\end{array}$ & CSR & $\mathbf{H}$ & CSR & $\mathbf{H}$ & $\mathbf{U}$ & $\mathbf{H}$ & $\mathbf{U}$ & $\mathbf{H}$ & CSR & $\begin{array}{c}\text { S1 } \\
\text { ORT }\end{array}$ & CSR & $\begin{array}{c}\text { T1 } \\
\text { ORT }\end{array}$ & CSR & CSR & $\begin{array}{c}\text { S2 } \\
\text { ORT }\end{array}$ & CSR & $\begin{array}{c}\text { T2 } \\
\text { ORT }\end{array}$ & $\mathbf{U}$ & $\begin{array}{c}\text { S3 } \\
\text { ORT }\end{array}$ & $\begin{array}{c}\text { T3 } \\
\text { ORT }\end{array}$ & $\mathbf{U}$ & $\begin{array}{c}\text { S4 } \\
\text { ORT }\end{array}$ & $\begin{array}{c}\text { T4 } \\
\text { ORT }\end{array}$ \\
\hline $\begin{array}{l}\text { C-L } \\
\text { Group }\end{array}$ & $\mathbf{U}$ & $\mathbf{H}$ & $\mathbf{U}$ & $\mathbf{H}$ & $\mathbf{U}$ & $\mathbf{H}$ & $\mathbf{U}$ & $\mathbf{H}$ & $\mathbf{U}$ & $\begin{array}{c}\text { S1 } \\
\text { OLT }\end{array}$ & $\mathbf{U}$ & $\begin{array}{c}\text { T1 } \\
\text { OLT }\end{array}$ & $\mathbf{U}$ & $\mathbf{U}$ & $\begin{array}{c}\text { S2 } \\
\text { OLT }\end{array}$ & $\mathbf{U}$ & $\begin{array}{c}\text { T2 } \\
\text { OLT }\end{array}$ & $\mathbf{U}$ & $\begin{array}{c}\text { S3 } \\
\text { OLT }\end{array}$ & $\begin{array}{c}\text { T3 } \\
\text { OLT }\end{array}$ & $\mathbf{U}$ & $\begin{array}{c}\text { S4 } \\
\text { OLT }\end{array}$ & $\begin{array}{c}\text { T4 } \\
\text { OLT }\end{array}$ \\
\hline $\begin{array}{l}\text { C-R } \\
\text { Group }\end{array}$ & $\mathbf{U}$ & $\mathbf{H}$ & $\mathbf{U}$ & H & $\mathbf{U}$ & $\mathbf{H}$ & $\mathbf{U}$ & $\mathbf{H}$ & $\mathbf{U}$ & $\begin{array}{c}\text { S1 } \\
\text { ORT }\end{array}$ & $\mathbf{U}$ & $\begin{array}{c}\text { T1 } \\
\text { ORT }\end{array}$ & $\mathbf{U}$ & $\mathbf{U}$ & $\begin{array}{c}\text { S2 } \\
\text { ORT }\end{array}$ & $\mathbf{U}$ & $\begin{array}{c}\text { T2 } \\
\text { ORT }\end{array}$ & $\mathbf{U}$ & $\begin{array}{c}\text { S3 } \\
\text { ORT }\end{array}$ & $\begin{array}{c}\text { T3 } \\
\text { ORT }\end{array}$ & $\mathbf{U}$ & $\begin{array}{c}\text { S4 } \\
\text { ORT }\end{array}$ & $\begin{array}{c}\text { T4 } \\
\text { ORT }\end{array}$ \\
\hline
\end{tabular}

Figure 1. Timeline of the experiment in post-natal days (PND). Rats undergoing the sleep deprivation had the manipulation implemented PND 2832 and 35-39, while control rats slept ad libitum. OLT: Object location task. ORT: Object recognition task. CSR-L: Chronic sleep restriction-OLT. CSR-R: Chronic sleep restriction-ORT. C-L: Control-OLT. C-R: Control-ORT. H: Habituation phase for task. S1: First study phase for task (adolescence). S2: Second study phase for task (adolescence). S3: Third study phase for task (adulthood). S4: Fourth study phase for task (adulthood). T1: First test phase for task (adolescence). T2: Second test phase for task (adolescence). T3: Third test phase for task (adulthood). T4: Fourth test phase for task (adulthood). U: Undisturbed sleep. 


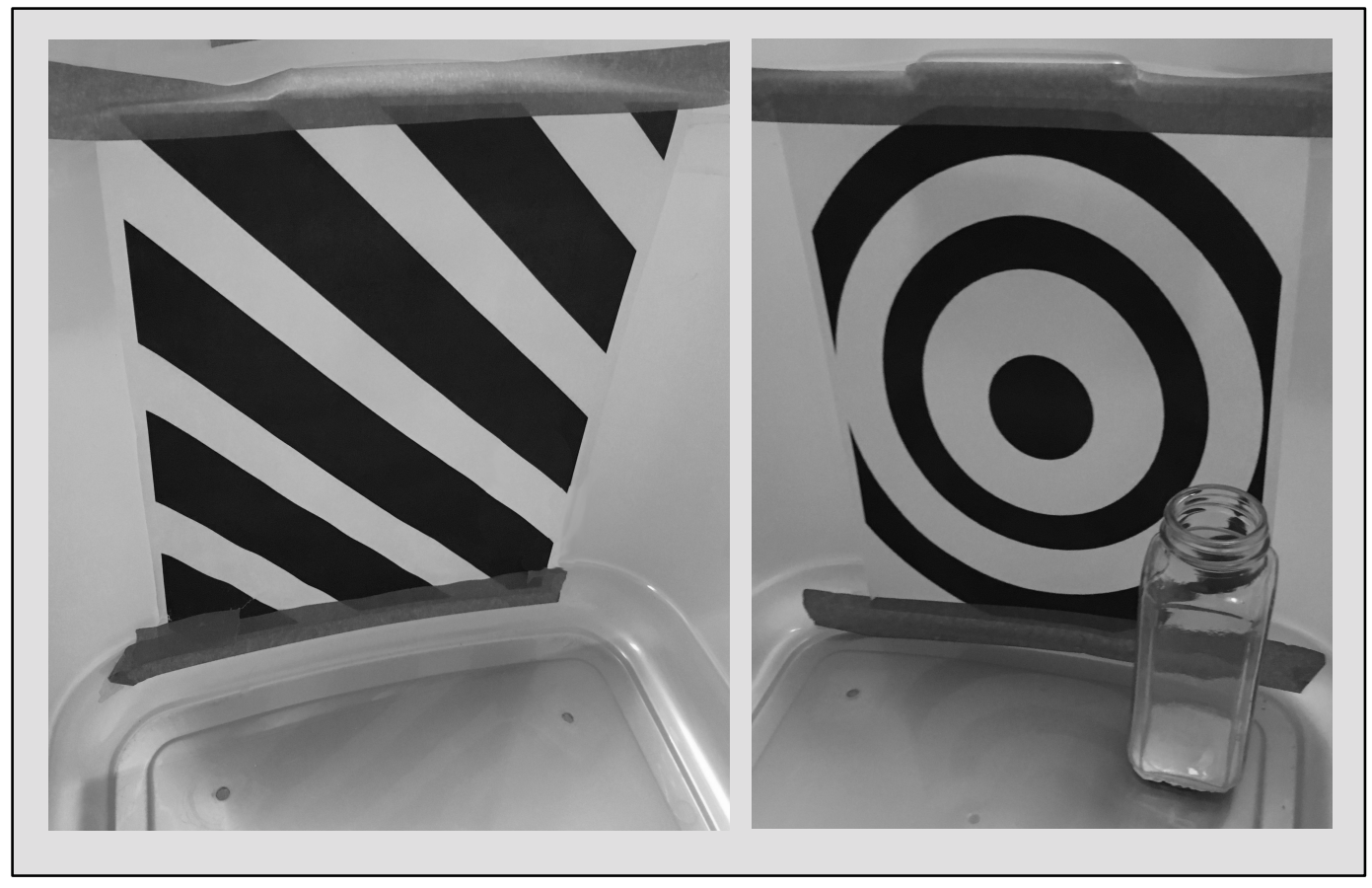

Figure 2. Pictures provided as spatial cues in the apparatus for the object location task. Photo by Kerry Howard.

The objects for the tasks were eight biologically meaningless items. A pilot study with eight adult rats was performed prior to the start of the main study to check that the rats showed no implicit biases for one (or more) object(s) over the others. An ethanol solution was used to clean the apparatus and objects of potential odor cues after every use.

Habituation phase. Subjects in all groups underwent habituation procedures over the course of four days, on PND 32, 33, 34, and 35 (Ennaceur and Delacour, 1988; Ngo et al., 2013) (Figure 1). During habituation, rats were placed in their respective (OLT versus ORT) experimental apparatus without any objects and allowed to freely explore for a 10-minute period immediately preceding the sleep phase on each of the consecutive days.

Study phase. The study phases began 24 hours after the completion of the final day of habituation (PND 36). During study phases, the rats had an opportunity to explore two identical objects placed in the back center of the apparatus (Ozawa et al., 2011). Over the five days of 
study phases and testing in adolescence, rats were exposed to both a 5 min study phase and a 20 min study phase, the order of which was counterbalanced. Half the subjects experienced the 5 min study phase on PND 36, while the second half experienced the 20 min study phase on that day, and the reverse occurred on PND 39. Rats also completed the study phase protocol as adults on PND 66 and 69 resulting in four total study phases each with novel identical object pairs.

Test phase. To examine long-term memory, testing occurred $24 \mathrm{hrs}$ after the respective study phase (Ozawa et al., 2011). Over the course of the experiment, each subject underwent the test phase on four separate occasions. In adolescence, rats were tested at PND 37 and PND 40 to examine immediate cognitive effects of CSR. In adulthood, rats were tested at PND 67 and PND 70 to examine long-term effects of CSR. Therefore, all rats were tested following $20 \mathrm{~min}$ and 5 min study phases in both adolescence and adulthood. A recovery period of $48 \mathrm{hrs}$ between the first test and second study phase, for each age period (PND 38 and 68), was implemented to prevent carryover effects from the first study phase. Between the conclusion of the sleep manipulation on PND 39 and the testing in adulthood, rats in both groups slept ad libitum.

The test phases differed based on cognitive task condition. In the OLT, the two objects in the apparatus were the same objects as in the study phase, but one object was moved to a novel location within the apparatus. In the ORT, one of the objects in the apparatus was the same as in the study phase, while the second object was a novel object placed in the same location as the prior object. The locations of the moved objects for the OLT and the novel object for the ORT were counterbalanced across the four test phases and rats to reduce the possibility that differences are due to the order of the these manipulations or place preferences. Figure 3 illustrates an example of setup differences between the study phase and test phase for the tasks. 


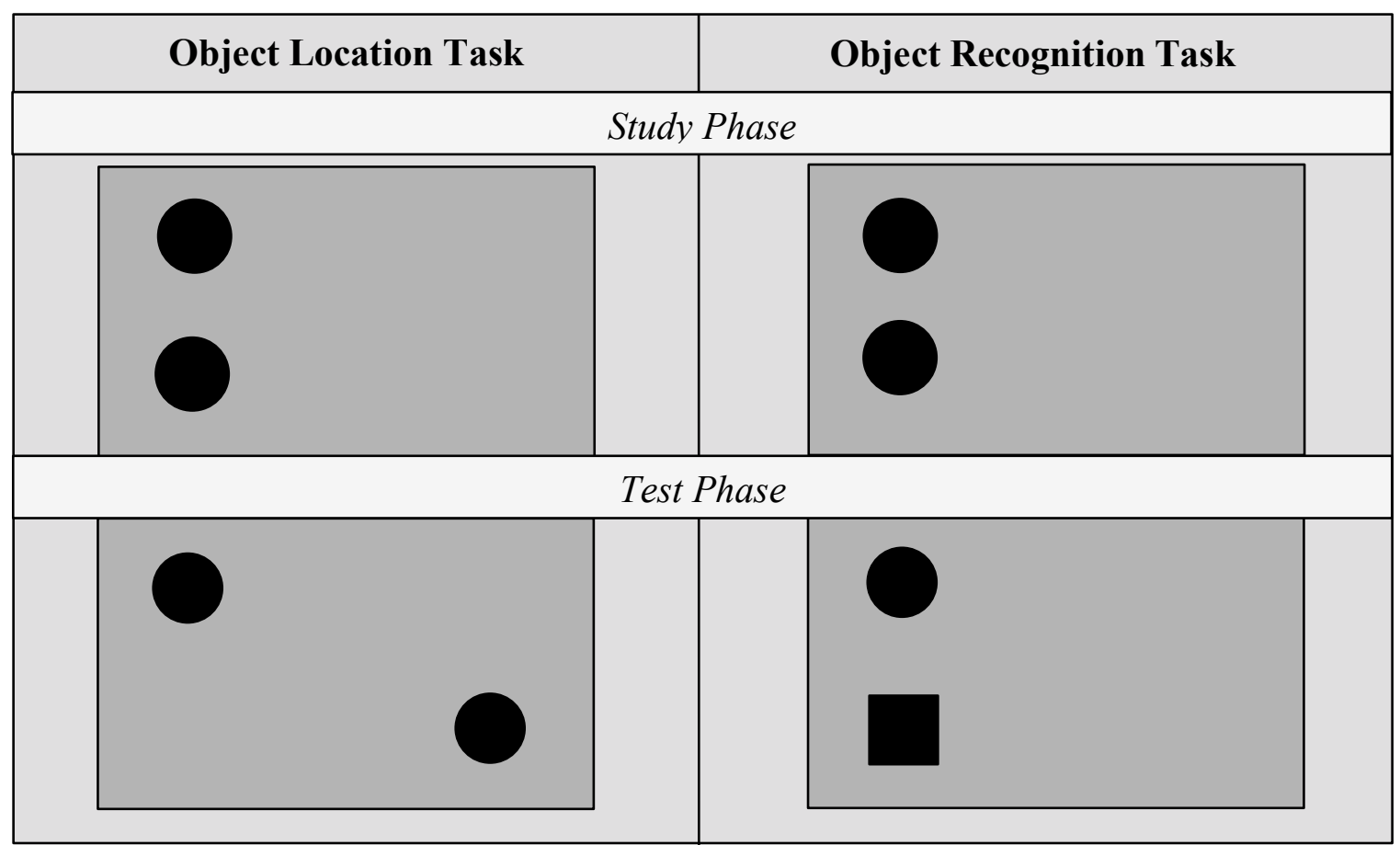

Figure 3. Example apparatus setup for study phase and test phase of the cognitive tasks. Location of the moved objects in the object location task (OLT) and novel object in the object recognition task (ORT) was counterbalanced across rats and test phases.

\section{Data Analysis}

Time spent spontaneously exploring an object was used as a measure of recognition of the object, with less exploration suggesting memory for the object (Binder et al., 2011; Ennaceur \& Delacour, 1988, Ngo et al., 2013; Palchykova, Crestani, et al., 2006; Palchykova, WinskySommerer et al., 2006). Exploration of an object was defined as touching the object with the nose or directing the nose $\leq 2 \mathrm{~cm}$ from the object (Ennaceur \& Delacour, 1988). Alternative interaction with the object, such as sitting on it or passing it, was not recorded as exploration, unless the subject simultaneously fulfilled the above requirements for exploration. We recorded exploration of the objects for two minutes at the start of the test phases, as the literature shows this is an important window for rats' display of preference for novelty (Dix \& Aggleton, 1999; Ozawa et al, 2011). 
As in prior OLT and ORT studies, differentiation between the two objects was calculated with a discrimination ratio and discrimination index (e.g., Binder et al., 2011; Dix \& Aggleton, 1999). The discrimination ratio (DR) gives a percentage of time spent exploring novelty out of total time spent exploring. It is calculated by dividing time spent exploring the novel item by time spent exploring the novel item added to time spent exploring the familiar item. The DR can be $>.5$ (preference for novelty), .5 (no preference), or $<.5$ (preference for familiarity). The discrimination index (DI) gives a measure of the difference in time spent exploring the two objects by subtracting the time spent exploring the familiar location/familiar object from the novel location/novel object (OLT/ORT). A positive DI indicates preference for the novel item over the familiar one, while a value of zero indicates no preference and a negative value indicates a preference for familiarity. While the DR and DI are both measures of novelty preference, we included both because they do not always produce identical results (e.g., Dix \& Aggleton, 1999). As a rat that remembers the study phase should prefer the new object or new object location, preference for novelty suggests that rat has intact long-term memory, while a preference for familiarity or no preference suggests that long-term memory is impaired or, in the case of the 5 min study phase, long-term memory was not established. The DR and DI were calculated for each test phase, averaged across subjects.

The videos of test phases were coded using Behavioral Observation Research Interactive Software (BORIS; Friard \& Gamba, 2016). For each test phase, we obtained a sum of the time that each rat spent exploring the familiar location/familiar object and exploring the novel location/novel object (OLT/ORT). These values were converted into DR and DI using the formulas above. The data was analyzed using IBM SPSS version 24. Two (one for DR and one for D1) 3-way mixed-measures ANOVAs were used to compare novelty and familiarity 
preferences for each cognitive task. The independent variables were study phase time (withinsubjects, $5 \mathrm{~min}$ and $20 \mathrm{~min}$ ), sleep condition (between-subjects, CSR and control), and age (within-subjects, adolescence and adulthood). Values of $p<.05$ were considered statistically significant. Post-hoc independent samples $t$-tests were utilized when there was evidence of an interaction. For the $t$-tests, a Bonferroni correction was used. We used partial eta squared as a measure of effect size for the ANOVAs (small $=.01$, medium $=.06$, large $=.14$ ) and Cohen's $d$ as a measure of effect size for post-hoc $t$-tests ( small $=.2$, medium $=.5$, large $=.8 ;$ Cohen, 1988). A quarter of the videos were randomly selected for inter-rater reliability assessment between two coders, with one coder blind to sleep condition and study phase time condition. A pre-set reliability criterion of correlation greater than .9 was set and met $(r=.935)$. 


\section{Results}

As reported in the following results, CSR appears to have negative consequences on long-term memory for spatial information in both adolescence and adulthood, but does not show evidence of affecting long-term recognition memory. A number of variables were examined to avoid confounds to the results. The present study began with a pilot study with adult rats to check for unconditioned biases for any of the objects that we intended to use. In a repeated measures design, all eight rats were exposed to each object in a variety of object pairing combinations. The results showed no evidence of preference for any object, as assessed by time spent exploring each one, $F(7,49)=0.63, p=.73, \eta_{p}^{2}=.08$. Additionally, given the counterbalancing of exposure to the 5 min study phase and the 20 min study phase, we examined the data for order effects. We found no evidence to suggest that exposure to the 5 min study phase first or the 20 min study phase first had an impact on the results in adolescence or adulthood.

\section{Object Location Task}

For the DR, the results showed an interaction between study time and sleep condition, $F(1,14)=16.75, p=.001, \eta_{p}^{2}=.55$. In the case of the 5 min study phase condition, follow-up tests showed that the CSR group $(M=.48, S D=.05)$ and control group $(M=.54, S D=.13)$ did not significantly differ, $t(9.104)=1.12, p=.29$, Cohen's $d=0.56$. The results from the 5 min study phase are in contrast to the 20 min phase condition in which the CSR group showed a preference for familiarity $(M=.45, S D=.13)$ and the control group showed a preference for novelty $(M=.69, S D=.12), t(14)=3.74, p=.002$, Cohen's $d=1.87$, suggesting that the CSR group had impaired memory for the novel object. The values around .5 for both groups in the 5 min study phase, indicating no preference for either object, show that 5 min was not sufficient to 
establish long-term memory. While the 20 min study phase was sufficient, the CSR group still did not show long-term memory, with value less than .5 .

Figure 4 illustrates these results, divided into adolescence (4A) and adulthood (4B). There was no evidence of a three-way interaction between study phase, sleep condition, and age $\left(F(1,14)=1.35, p=.27, \eta_{p}^{2}=.09\right.$; therefore, the significant 2-way interaction between study phase and sleep time without age suggests a similar pattern in adolescence and adulthood, as shown in Figure 4. As such, the study phase by sleep condition pattern was mimicked in adulthood: there were similar scores for CSR group $(M=.56, S D=.17)$ and the control group $(M$ $=.51, S D=.18)$ in the 5 min study phase, $t(14)=-0.54, p=.60$, Cohen's $d=-0.27$. In the $20 \mathrm{~min}$ study phase, the CSR group showed a preference for familiarity $(M=.42, S D=.09)$, while the control group showed a preference for novelty $(M=.68, S D=.06), t(14)=7.01, p<.001$, Cohen's $d=3.50$. There was also a significant main effect of sleep condition, $F(1,14)=10.09, p$ $=.007, \eta_{p}^{2}=.42$. The CSR group showed no preference $(M=.48, S D=.08)$ while the control group $(M=.61, S D=.08)$ showed a preference for novelty across the study phase time and age variables. There was no evidence of additional two-way interactions between age and study time $\left(F(1,14)=0.68, p=.42, \eta_{p}^{2}=.05\right)$ or between age and sleep condition $(F(1,14)=0.60, p=.45$, $\left.\eta_{p}^{2}=.04\right)$. There was also no evidence of a main effect of age $\left(F(1,14)=0.06, p=.80, \eta_{p}^{2}=.005\right)$ or a main effect of study phase $\left(F(1,14)=1.60, p=.23, \eta_{p}^{2}=.10\right)$.

A similar pattern of results was seen in the case of DI. Again, the results showed an interaction between study phase time and sleep condition, $F(1,14)=16.51, p=.001, \eta_{p}^{2}=.54$, such that the CSR group $(M=-2.09, S D=4.38)$ and control group $(M=1.78, S D=7.52)$ did not significantly differ in the 5 min study phase, $t(14)=1.26, p=.23$, Cohen's $d=0.63$; but the CSR group $(M=-4.10, S D=6.82)$ showed a preference for familiarity and the control group $(M=$ 
$11.57, S D=8.63)$ showed a preference for novelty in the 20 min study phase, $t(14)=4.03, p=$ .001 , Cohen's $d=2.02$. Again, there was no evidence of a three-way interaction between study phase, sleep condition, and age $\left(F(1,14)=1.98, p=.18, \eta_{p}^{2}=.12\right)$. In adulthood, the CSR group showed a higher average DI in the 5 min study phase $(M=4.50, S D=14.38)$ compared to the control group $(M=0.43, S D=10.29)$, but this difference was not statistically significant, $t(14)=$ $-0.65, p=.53$, Cohen's $d=-0.33$. In the 20 min study phase, the CSR group $(M=-5.89, S D=$ 7.16) showed a familiarity preference with the control group showing a novelty preference ( $M=$ $14.39, S D=6.50), t(14)=5.93, p<.001$, Cohen's $d=2.97$. Also similarly to the DR, there was a main effect of sleep condition when measured with DI, with the CSR group lower $(M=-1.90$, $S D=5.03)$ than the control group $(M=7.04, S D=5.03), F(1,14)=12.61, p=.003, \eta_{p}^{2}=.47$. There was no evidence of an interaction between age and study time $\left(F(1,14)=0.23, p=.64, \eta_{p}^{2}\right.$ $=.02)$ or between age and sleep condition $\left(F(1,14)=0.27, p=.61, \eta_{p}^{2}=.02\right)$. There was also no evidence of a main effect of age $\left(F(1,14)=0.94, p=.35, \eta_{p}^{2}=.06\right)$ or a main effect of study phase $\left(F(1,14)=1.63, p=.22, \eta_{p}^{2}=.10\right)$. 


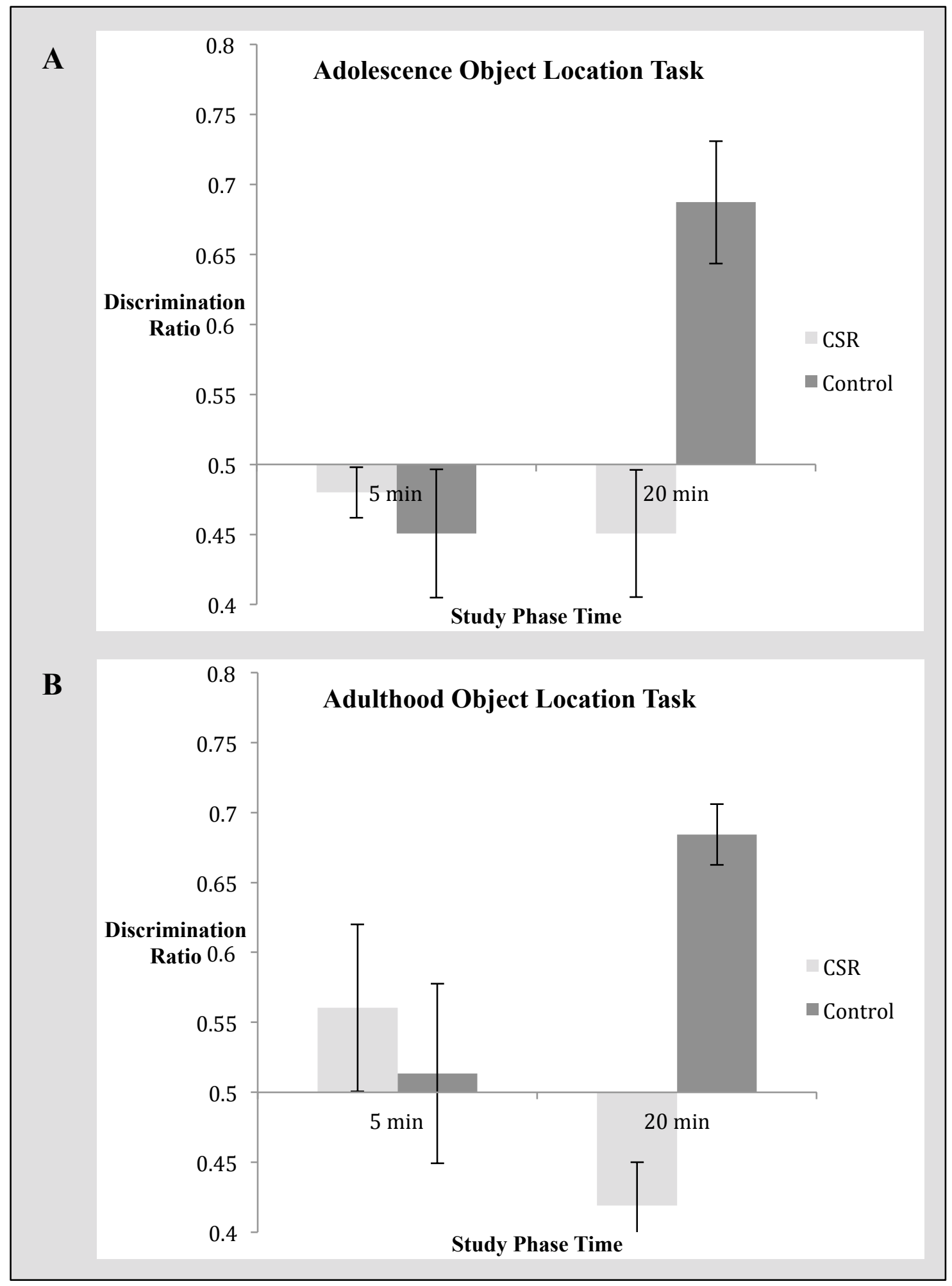

Figure 4. Discrimination ratio results for the adolescent (A) and adulthood (B) object location task. Above $.5=$ novelty preference, $.5=$ no preference, below $.5=$ familiarity preference. Data are presented as mean \pm standard error. $N=8$ per group. 


\section{Object Recognition Task}

For the DR ANOVA, there was a main effect of age, such that adults showed higher DR $(M=.69, S D=.07)$ than adolescents $(M=.57, S D=.06), F(1,14)=13.99, p=.002, \eta_{p}^{2}=.50$.

Beyond this, there were no significant results. There was no evidence of a three-way interaction, $F(1,14)=1.08, p=.32, \eta_{p}^{2}=.07$. There was also no evidence of two-way interactions between age and study time $\left(F(1,14)=0.10, p=.75, \eta_{p}^{2}=.007\right)$, age and sleep condition $(F(1,14)=2.26$, $\left.p=.16, \eta_{p}^{2}=.14\right)$, or sleep condition and study phase $\left(F(1,14)=0.28, p=.60, \eta_{p}^{2}=.02\right.$. Finally, there was no evidence of a main effect of sleep condition $\left(F(1,14)=1.52, p=.24, \eta_{p}^{2}=.10\right)$ or study time $\left(F(1,14)=2.46, p=.14, \eta_{p}^{2}=.15\right)$. These results are shown in Figure 5 .

Furthermore, the DI mimicked the results for the DR. A main effect of age was observed, $F(1,14)=7.06, p=.019, \eta_{p}^{2}=.34$. Adult rats showed higher DI $(M=17.52, S D=7.55)$ than the adolescent rats $(M=7.75, S D=8.08)$. However, there was no evidence of a three-way interaction, $F(1,14)=1.54, p=.24, \eta_{p}^{2}=.10$. There was also no evidence of two-way interactions between age and study time $\left(F(1,14)=0.53, p=.48, \eta_{p}^{2}=.04\right)$, age and sleep condition $\left(F(1,14)=1.22, p=.29, \eta_{p}^{2}=.08\right)$, or sleep condition and study phase $(F(1,14)=$ $\left.0.002, p=.97, \eta_{p}^{2}=.000\right)$. There was also no evidence of a main effect of sleep condition $\left(F(1,14)=0.94, p=.35, \eta_{p}^{2}=.06\right)$ or study time $\left(F(1,14)=1.84, p=.20, \eta_{p}^{2}=.12\right)$ 


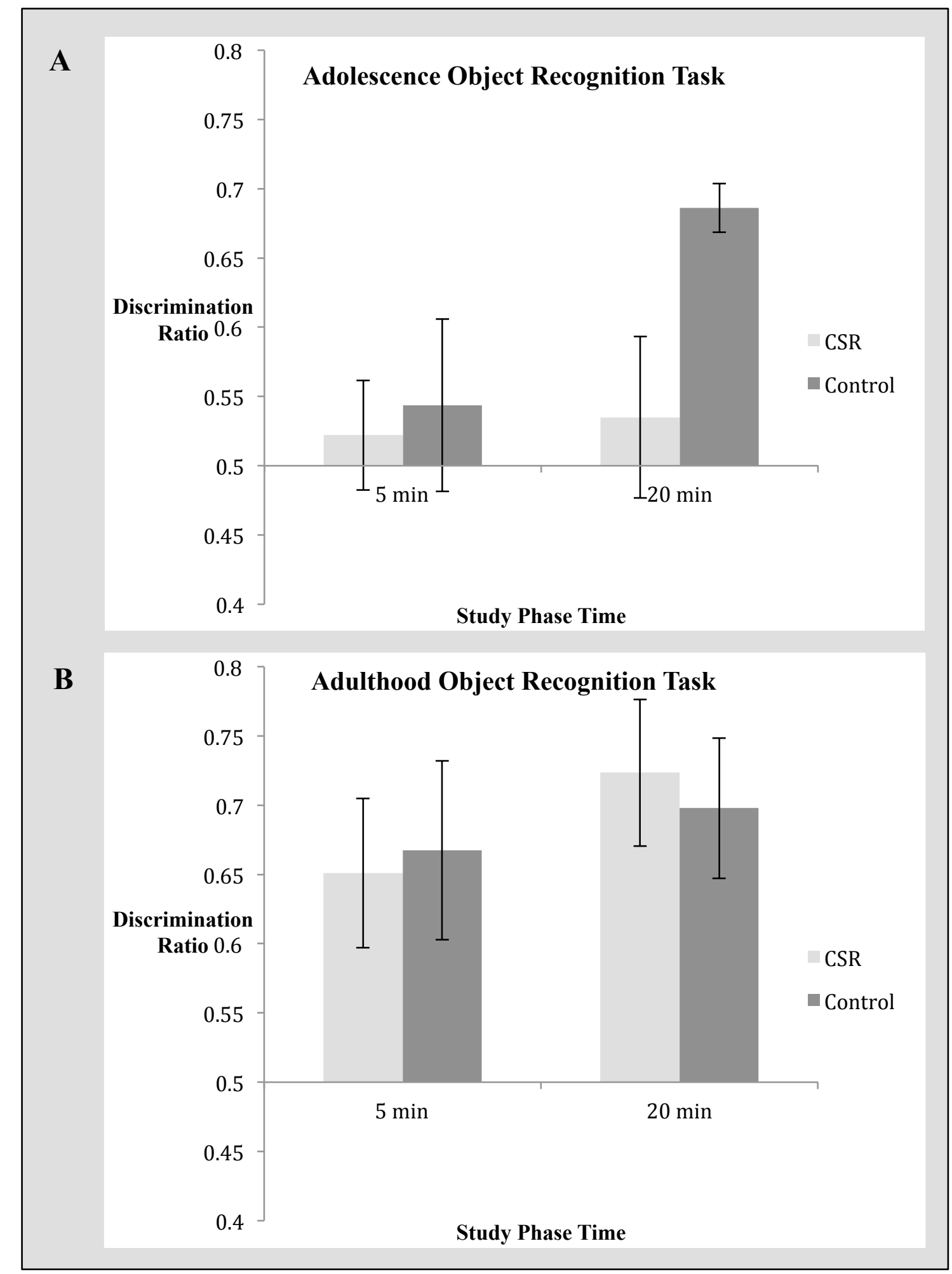

Figure 5. Discrimination ratio results for the adolescent (A) and adulthood (B) object recognition task. Above $.5=$ novelty preference, $.5=$ no preference, below $.5=$ familiarity preference. Data are presented as mean \pm standard error. $N=8$ per group. 


\section{Discussion}

The present study used rats to model the CSR experienced by adolescent humans to investigate the potential cognitive consequences of CSR during this crucial developmental period. The results demonstrated that in measures of spatial memory, CSR impairs long-term memory in adolescence and that the effects are long-lasting. Given the appropriate circumstances to establish a long-term memory, rats that experienced CSR showed long-term memory impairment in adolescence, unable to distinguish between an object in a familiar location and an object in a novel location during the OLT. The negative effect of CSR was also observed with continued impairment in long-term memory when the same rats were tested in adulthood. The long-lasting consequences were demonstrated by the lack of a significant effect of age in the OLT, indicating that the pattern of impairment was consistent between the adolescent period and the adulthood period. These consequences were not observed for non-spatial memory. That is, there was no evidence of significant effects of CSR on long-term memory in the ORT.

We hypothesized that CSR, implemented for four hours per day, excluding a two-day "weekend," throughout the two-week adolescent period, would impair long-term memory abilities for both hippocampal dependent and non-hippocampal dependent memory. A 5 min study time is not enough to establish a long-term memory, thus we hypothesized that subjects would not show long-term memory at test 24 hours later (Ozawa et al., 2011). This result was observed in the OLT, confirming that we tapped into long-term memory mechanisms. In contrast to the 5 min study phase, the 20 min study phase was sufficient to establish a long-term memory and we therefore hypothesized that CSR rats would show long-term memory impairment. Our hypothesis was supported in the case of hippocampal dependent memory. That is, subjects that were exposed to CSR in adolescence demonstrated long-term memory impairment in an object 
location task. Additionally, the pattern of impairment was displayed in adulthood, demonstrating the long-lasting consequences of CSR during the developmental period of adolescence.

On the other hand, the test for non-hippocampal dependent memory with the ORT contradicted our hypothesis. As there was no precedent in the literature for the effects of sleep restriction in adolescence on non-hippocampal dependent memory, our hypothesis for this task was based on indirectly related prior literature. That is, Hairston and colleagues (2005) showed that non-hippocampal dependent memory was not impaired following CSR in adulthood. However, while Yang and colleagues (2012) used a hippocampal dependent task, their results demonstrated increased vulnerability in adolescence to CSR, compared to adults. The results of the present study are consistent with the lack of effect of CSR on non-hippocampal dependent memory observed by Hairston and colleagues and partially consistent with the findings of Yang and colleagues. The present results did not extend the vulnerability demonstrated by Yang and colleagues to CSR in adolescence on a non-hippocampal dependent memory task, but both studies showed an effect of age such that adolescents performed worse than adults. The present study's findings of the ORT showed that CSR in adolescence did not have immediate or longlasting cognitive consequences to non-spatial long-term memory, impairing hippocampal dependent and non-hippocampal dependent memory differently.

The unexpected lack of impairment in non-hippocampal dependent memory provides the benefit of informing the findings in the hippocampal dependent task. Namely, the non-significant results suggest that the significant results in the hippocampal dependent task are not the product of the CSR manipulation causing motor impairment or debilitating stress. As a result, the findings in the hippocampal dependent task represent long-term memory impairment, unexplained by confounding potential effects of CSR. 


\section{Implications}

The findings in this study are important because they suggest that the CSR that is common to adolescents can have a negative effect on their cognitive abilities. From a practical standpoint, this provides evidence that sleep patterns may influence less than optimal performance among adolescents across various domains, from academics to safe driving (Danner \& Phillips, 2008). Therefore, from a perspective concerned with healthy, safe, and optimal adolescent development, the importance of these results cannot be overstated.

Perhaps the most critical and disturbing aspect of this study is the finding that the impairment to long-term memory is also evident after four weeks of undisturbed sleep following the CSR manipulation. As our hypothesis was supported in the case of hippocampal dependent memory in adulthood as well as adolescence, the findings suggest that CSR may interrupt normal adolescent cognitive development, resulting in chronic impairment. That is, despite the opportunity to recover sleep over an undisturbed four weeks, CSR made an impression in adolescence that persists. Such a conclusion is corroborated by neurological evidence. Bellesi and colleagues (2018) found that chronic sleep loss in adolescent mice negatively affected myelination processes, resulting in decreased myelin thickness. Adolescence is a critical period for essential neurological changes, including myelin production (Spear, 2013). As a result, the findings of the present study, along with those of Bellesi and colleagues, suggest that the neurological advancement that is meant to occur in adolescence may be stunted by adolescent CSR such that the brain is restricted from developing adult-type neurological maturation. As with the adolescence findings, these results are important to the insurance of healthy adolescent development, especially with the concern that unhealthy adolescent development can haunt into adulthood. 
Finally, the results suggest that non-hippocampal dependent memory may be more resistant to sleep deprivation than hippocampal dependent memory. The non-hippocampal dependent and hippocampal dependent distinction has been observed in the literature (see McCoy \& Strecker, 2011, for review). As noted, Hairston and colleagues (2005) found that CSR impaired hippocampal dependent, but not non-hippocampal dependent memory. Additionally, this finding is observed in alternative sleep deprivation procedures (see McCoy \& Strecker, 2011, for review). For example, selective REM deprivation has been shown to impaired spatial memory rather than non-spatial memory (e.g., Youngblood, Zhou, Smagin, Ryan, \& Harris, 1997; Walsh et al., 2011). The differentiation reaffirms the necessity for research to examine both hippocampal dependent and non-hippocampal dependent memory, such that the distinction can continue to be examined.

\section{Limitations and Future Directions}

Future studies should re-examine non-hippocampal dependent memory by improving on limitations to this study that may inform the unexpected results. Primarily, variability among the rats in the non-hippocampal dependent task in adolescence was substantial and may have presented a problem for obtaining statistical significance. Specifically, we revisited videos in which the rats showed the greatest deviation from expected results and examined patterns among the data. Despite the fact that we did a pilot study with different rats to ensure no unconditioned object preferences, it appears that three rats showed an unconditioned preference for one of the objects used in the non-hippocampal dependent task. That is, the rats attended to this object more so than the other object regardless of whether the object was novel or familiar. The results of the pilot study did not suggest that rats preferred this object. A possible reason for this difference is that the pilot study utilized adult rats and perhaps adolescent rats show differential implicit object 
preferences. Additionally, the adult rats that were used in the pilot study had been used in other experiments in the laboratory, while the adolescent rats used in this study were naïve to any experimental procedures prior to this study. There were no other observed systematic differences that would account for the variability.

The non-significant results in the ORT may also be the product of differences in the difficulty of the OLT and ORT. Ozawa and colleagues (2011) conducted the experiment on which the 5 min study phase versus 20 min study phase hypothesis was based; however, they only tested the OLT. It is possible that this distinction in study phase times does not apply to the ORT. That is, we may have been tapping into something other than long-term memory mechanism or alternative study phase times may be required for the ORT. It is also possible that the OLT is more difficult than the ORT, although there is no evidence in the literature of a direct comparison. If the OLT is more difficult, it would likely require a longer study phase time to establish long-term memory, while the rats may be able to perform the ORT regardless of study phase time or sleep quantity. The results from Palchykova, Winsky-Sommerer, and colleagues (2006) inform this idea, as even undisturbed control mice were unable to differentiate, after a 24 hour delay, between an object in a novel location and an object in a familiar location following five 5 min study sessions (25 total study phase minutes). As a result, the lack of significant impairment as a function of CSR for the ORT may be because the task was easy enough that even the CSR group was able to complete it successfully. Future studies should compare necessary study phase times for the OLT and ORT to investigate if one requires a longer study phase to establish a long-term memory, thereby indicating a higher level of difficulty.

The results of the present study yield a number of novel ideas about the nature of adolescent sleep deprivation. In particular, the adulthood results produce a critical new question: 
is there potential for recovery from the long-term memory impairment caused by CSR in adolescence? Testing beyond the four-week mark employed in this study would inform the theory that adolescent CSR interrupts adult-type maturation. That is, while our study shows that the impairment persists into adulthood, future studies could examine at what age, if ever, the impairment recedes, thereby speaking to cognitive recovery outlook.

Additionally, research should examine the relationship between quantity and quality of adolescent sleep. The present study focused on quantity, implementing a sleep manipulation that deprived the rats of hours of sleep, as is the nature of research on the effects of CSR. However, it may not only be the quantity of sleep that is important, but the quality of sleep as well. In human adolescents, insufficient sleep results in compensatory changes in sleep, such as daytime sleepiness (e.g., sleeping during class) and oversleeping on weekends (Beebe et al., 2010; Crowley et al., 2014; Owens et al., 2010; Wahlstrom, 2002). Yang and colleagues (2012) investigated sleep rebound on weekends in rats and during non-deprivation hours. They found evidence of increased sleep in these periods, with particular rebound of REM sleep. However, despite some sleep rebound, there were still effects of CSR in the adolescent population. Therefore, does the compensatory quantity of sleep that adolescents may demonstrate satisfy quality needs in order to sufficiently remedy the impairment from CSR? Answering these questions would provide details of adolescent sleep needs, thereby improving potential interventions aimed at reducing adolescent CSR.

\section{Conclusions}

This study addresses a number of outstanding questions in the sleep deprivation literature. First, CSR is rarely used to examine the effects of sleep deprivation and, while a number of alternative forms of sleep deprivation have been used to model sleep impairments, 
CSR has been utilized only once as a model of limited adolescent sleep, measuring resultant cognitive impairment (Yang et al., 2012). Second, the present study is the first to use the OLT and ORT to measure the effects of CSR. The results show that the OLT, at least, can provide information regarding hippocampal dependent memory following CSR. However, more research is necessary to determine if the non-significant results of the ORT represent unaffected nonhippocampal dependent memory or a lack of sensitivity of the ORT to the effects of CSR. Finally, while Yang and colleagues (2012) investigated the effect of CSR on both adolescents and adults, their study was not longitudinal in nature. Therefore, the present study is the only one thus far to show that CSR in adolescence can negatively affect cognition immediately and that impairments can persist into adulthood.

While an appropriate quantity of sleep is particularly important in adolescence, adolescent students experience CSR due to a change in sleep patterns with persistently early wake times for school. The results of this study have practical suggestions relative to adolescent health and educational policy. For many years, high schools have debated implementing later start times. Based on the idea that adolescents are not getting enough sleep, researchers investigated the effects of starting school later (Owens et al., 2010). In one study, a simple 30minute delay resulted in 45 more minutes of sleep per night and increased the percent of students getting enough sleep every night from less than $20 \%$ to over $50 \%$. Additionally, while at least 8 hours is recommended for adolescents, the delay resulted in only $7 \%$ of students getting less than 7 hours of sleep, compared to 34\% prior to the modification (Hirshkowitz et al., 2015; Owens et al., 2010). Thus, later start times reduce adolescent CSR, resulting in a number of positive outcomes including improved mood, decreased absences or tardies, decreased sleepiness in class, improved academic achievement, and decreased teen car accidents (Carrell, Maghakian, \& West, 
2011; Danner \& Phillips, 2008; Owens et al., 2010; Wahlstrom, 2002). Despite these findings, a debate persists about the necessity to make start time changes on a widespread scale (Owens et al., 2010). For the optimal and long-lasting health and cognitive wellbeing of adolescents, adjustments that reduce the prevalence of CSR among this population in today's society need to be considered. 


\section{References}

Agostini, A., Carskadon, M.A., Dorrian, J., Coussens, S., \& Short, M.A. (2016). An experimental study of adolescent sleep restriction during a simulated school week: Changes in phase, sleep staging, performance and sleepiness. Journal of Sleep Research, 26, 227-235. https://doi.org/10.1111/jsr.12473.

Akirav, I. \& Maroun, M. (2006). Ventromedial prefrontal cortex is obligatory for consolidation and reconsolidation of object recognition memory. Cerebral Cortex, 16, 1759-1765.

Balderas, I., Rodriguez-Ortiz, C.J., Salgado-Tonda, P., Chavez-Hurtado, J., McGaugh, J.L., Bermudez-Rattoni, F. (2008). The consolidation of object and context recognition memory involve different regions of the temporal lobe. Learning \& Memory, 15, 618624.

Bava, S. \& Tapert, S.F. (2010). Adolescent brain development and the risk for alcohol and other drug problems. Neuropsychology Review, 20, 398-413. https://doi.org/10.1007/s11065010-9146-6.

Beebe, D.W. (2011). Cognitive, behavioral, and functional consequences of inadequate sleep in children and adolescents. Pediatric Clinics of North America, 58, 649-665. https://doi.org/10.1016/j.pcl.2011.03.002.

Beebe, D.W., Rose, D., \& Amin, R. (2010). Adolescent health brief: Attention, learning, and arousal of experimentally sleep-restricted adolescents in a simulated classroom. Journal of Adolescent Health, 47, 523-525. https://doi.org/10.1016/j.jadohealth.2010.03.005.

Bellesi, M., Haswell, J.D., de Vivo, L., Marshall, W., Roseboom, P.H., Tononi, G., \& Cirelli, C. (2018). Myelin modifications after chronic sleep loss in adolescent mice. Sleep, zsy034. http://doi.org/10/1093/sleep/zsy034. 
Billeh, Y.N., Rodriguez, A.V., Bellesi, M., Bernard, A., de Vivo, L., Funk, C.M., ... Tononi, G. (2016). Effects of chronic sleep restriction during early adolescence on the adult pattern of connectivity of mouse secondary motor cortex. eNeuro, 3, 1-15. https://doi.org/10.1523/ENEURO.0053-16.2016.

Binder, S., Baier, P. C., Mölle, M., Inostroza, M., Born, J., \& Marshall, L. (2012). Sleep enhances memory consolidation in the hippocampus-dependent object-place recognition task in rats. Neurobiology of Learning and Memory, 97, 213-219. https://doi.org/10.1016/j.nlm.2011.12.004.

Bonnet, M.H. \& Arand, D.L. (1995). We are chronically sleep deprived. Sleep, 18, 908-911. Brenhouse, H.C. \& Andersen, S.L. (2011). Developmental trajectories during adolescence in males and females: a cross-species understanding of underlying brain changes. Neuroscience \& Biobehavioral Reviews, 35(8), 1687-1703. https://doi.org/10.1016/j.neubiorev.2011.04.013.

Capellini, I., Barton, R.A., McNamara, P., Preston, B.T., \& Nunn, C.L. (2008). Phylogenetic analysis of the ecology and evolution of mammalian sleep. Evolution, 62, 1764-1776. https://doi.org/10.1111/j.1558-5747.2008.00392.x.

Carrell, S.E., Maghakian, T., \& West, J.E. (2011). A's from Zzzz's? The causal effect of school start time on the academic achievement of adolescents. American Economic Journal: Economic Policy, 3(3), 62-81.

Carskadon, M.A. (2011). Sleep in adolescents: The perfect storm. Pediatric Clinics of North America, 58, 637-647. https://doi.org/10.1016/j.pcl.2011.03.003. 
Carskadon, M.A. \& Dement, W.C. (2011). Monitoring and staging human sleep. In M.H. Kryger, T. Roth, \& W.C. Dement (Eds.), Principles and practice of sleep medicine, $5^{\text {th }}$ edition (pp. 16-26). St. Louis: Elsevier Saunders.

Cohen, J. (1988). Statistical power analysis for the behavioral sciences (2nd ed.). Hillsdale, NJ: Lawrence Erlbaum Associates.

Córdova, C.A., Said, B.O., McCarley, R.W., Baxter, M.G., Chiba, A.A., \& Strecker, R.E. (2006). Sleep deprivation in rats produces attention impairments on a 5-Choice Serial Reaction Time Task. Sleep, 29, 69-76.

Crowley, S.J., Van Reen, E., LeBourgeois, M.K., Acebo, C., Tarokh, L., Seifer, R., .. . Carskadon, M.A. (2014). A longitudinal assessment of sleep timing, circadian phase, and phase angle of entrainment across human adolescence. PloS ONE, 9, 1-11. https://doi.org/10.1371/journal.pone.0112199.

Czéh, B., \& Lucassen, P.J. (2007). What causes the hippocampal volume decrease in depression? Are neurogenesis, glial changes and apoptosis implicated? European Archives of Psychiatry and Clinical Neuroscience, 257, 250 -260. https://doi.org/10.1007/s00406007-0728-0.

Danner, F. \& Phillips, B. (2008). Adolescent sleep, school start times, and ten motor vehicle crashes. Journal of Clinical Sleep Medicine, 4(6), 533-535.

Dix, S.L. \& Aggleton, J.P. (1999). Extending the spontaneous preference test of recognition: Evidence of object-context recognition. Behavioural Brain Research, 99, 191-200.

D’Hooge, D. \& De Deyn, P.P. (2001). Applications of the Morris water maze in the study of learning and memory. Brain Research Reviews, 36, 60-90. 
Ennaceur, A. \& Delacour, J. (1988). A new one-trial test for neurobiological studies of memory in rats. 1: Behavioral data. Behavioral Brain Research, 31, 47-59.

Ennaceur, A., Neave, N., \& Aggleton, J.P. (1997). Spontaneous object recognition and object location memory in rats: The effects of lesions in the cingulate cortices, the medial prefrontal cortex, the cingulum bundle and the fornix. Experimental Brain Research, 113, 509-519.

Eriksson, J., Vogel, E.K., Lansner, A., Bergström, F., \& Nyberg, L. (2015). Neurocognitive architecture of working memory. Neuron, 88(1), 33-46. https://doi.org/10.1016/j.neuron.2015.09.020.

Frank, M.G. (2006). The mystery of sleep function: Current perspectives and future directions. Reviews in Neuroscience, 17, 375-392.

Friard, O. \& Gamba, M. (2016). BORIS: A free, versatile open-source event-logging software for video/audio coding and live observations. Methods in Ecology and Evolution, 7, 1325-1330. https://doi.org/10.1111.2041-210X.12584.

Global Burden of Disease 2015 Mortality and Cause of Death Collaborators. (2016). Global, regional, and national life expectancy, all-cause mortality, and cause-specific mortality for 249 causes of death, 1980-2015: A systematic analysis for the Global Burdon of Disease Study 2015. Lancet, 388, 1459-1544. https://doi.org/10.1016/S0140-6738.

Guan, Z. Peng, X. \& Fang, J. (2004) Sleep deprivation impairs spatial memory and decreases extracellular signal-related kinase phosphorylation in the hippocampus. Brain Research, 1018, 38-47. https://doi.org/10.1007/s00406-007-0728-0.

Hagenauer, M.H. \& Lee, T.M. (2013). Adolescent sleep patterns in humans and laboratory animals. Hormones and Behavior, 64, 270-279. 
https://doi.org/10.1016/j.yhbeh.2013.01.013.

Hagewoud, R., Havekes, R., Novati, A., Keijser, J.N., van der Zee, E.A., \& Meerlo, P. (2010). Sleep deprivation impairs spatial working memory and reduces hippocampal AMPA receptor phosphorylation. Journal of Sleep Research, 19, 280-288. https://doi.org/10.1111/j.1365-2869.2009.00799.x.

Hairston, I.S., Little, M.T.M., Scanlon, M.D., Barakat, M.T., Palmer, T.D., Sapolsky, R.M., \& Heller, H.C. (2005). Sleep restriction suppresses neurogenesis induced by hippocampusdependent learning. Journal of Neurophysiology, 94, 4224-4233. https://doi.org/10.1152/jn.00218.2005.

Harker, K.T. \& Whishaw, I.Q. (2002). Place and matching-to-place spatial learning affected by rat inbreeding (Dark-Agouti, Fischer 344) and albinism (Wistar, Sprague-Dawley) but not domestication (wild rat vs. Long-Evans, Fischer-Norway). Behavioural Brain Research, 134, 467-477. https://doi.org/10.1016/S0166-4328(02)00083-9.

Hirshkowitz, M., Whiton, K., Albert, S.M., Alessi, C., Bruni, O., DonCarlos, L., . . Hillard, P.J.A. (2015). National Sleep Foundation's sleep time duration recommendations: Methodology and results summary. Sleep Health, 1, 40-43. https://doi.org/10.1016/j.sleh.2014.12.010.

Jiang, F., VanDyke, R.D., Zhang, J., Li, F., Gozal, D., \& Shen, X. (2011). Effect of chronic sleep restriction on sleepiness and working memory in adolescents and young adults. Journal of Clinical and Experimental Neuropsychology, 33(8), 892-900. http://doi.org/10.1080/13803395.2011.570252.

Kim, Y., Laposky, A.D., Bergmann, B.M., \& Turek, F.W. (2007). Repeated sleep restriction in rats leads to homeostatic and allostatic responses during recovery sleep. PNAS, 104, 
10697-10702. https://doi.org/10.1073 pnas.0610351104.

Leemburg, S., Vyazovskiy, V.V., Olcese, U., Bassetti, C.L., Tononi, G., \& Cirelli, C. (2010). Sleep homeostasis in the rat is preserved during chronic sleep restriction. PNAS, 107, 15939-15944. https://doi.org/10.1073/pnas.1002570107.

Lenroot, R.K. \& Giedd, J.N. (2006). Brain development in children and adolescents: Insights from anatomical magnetic resonance imaging. Neuroscience and Biobehavioral Reviews, 30, 718-729. https://doi.org/10.1016/j.neubiorev.2006.06.001.

Lui, Y., Wheaton, A.G., Chapman, D.P., Cunningham, T.J., Lu, H., \& Croft, J.B. (2016).

Prevalence of healthy sleep duration among adults - United States, 2014. MMWR, 65, 137-141. http://doi.org/10.15585/mmwr.mm6506a1.

McCoy, J.G., Christie, M.A., Kim, Y., Brennan, R., Poeta, D.L., McCarley, R.W., \& Strecker, R.E. (2013). Chronic sleep restriction impairs spatial memory in rats. NeuroReport, 24 , 91-95. https://doi.org/10.1097/WNR.0b013e32835cd97a.

McCoy, J.G. \& Strecker, R.E. (2011). The cognitive cost of sleep lost. Neurobiology of Learning and Memory, 96, 564-582. https://doi.org/10.1016/j.nlm.2011.07.004.

Morrell, M.J., McRobbie, D.W., Quest, R.A., Cummin, A.R., Ghiassi, R., \& Corfield, D.R. (2003). Changes in brain morphology associated with obstructive sleep apnea. Sleep Medicine, 4, 451-454. https://doi.org/10.1016/S1389-9457(03)00159-X.

Morris, R. (1984). Developments of a water-maze procedure for studying spatial learning in the rat. Journal of Neuroscience Methods, 11, 47-60.

Mumby, D.G., Pinel, J.P.J., \& Wood, E.R. (1990). Nonrecurring-items delayed nonmatching-tosample in rats: A new paradigm for testing nonspatial working memory. Psychobiology, 18, 321-326. https://doi.org/10.3758/BF03327250. 
Nagai, M., Hoshide, S., \& Kario, K. (2010). Sleep duration as a risk factor for cardiovascular disease-A review of the recent literature. Current Cardiology Review, 6, 54-61. https://doi.org/10.2174/157340310790231635.

National Sleep Foundation. (2006). 2006 sleep in America poll summary findings. Retrieved from http://sleepfoundation.org/sites/default/files/2006_summary_of_findings.pdf.

Ngo, C.T., Lloyd, M., \& Hunter, A. (2013, March). Varying Effects of REM deprivation on object recognition and object location tasks. Poster session presented at the meeting of the American Psychological Association, New York, NY.

Novati, A., Hulshof, H.J., Koolhaas, J.M., Lucassen, P.J., \& Meerlo, P. Chronic sleep restriction causes a decrease in hippocampal volume in adolescent rats, which is not explained by changes in glucocorticoid levels or neurogenesis. Neuroscience, 190, 145-155. https://doi.org/10.1016/j.neuroscience.2011.06.027.

O'Brien, N., Lehmann, H., Lecluse, V., \& Mumby, D.G. (2006). Enhanced context-dependency of object recognition in rats with hippocampal lesions. Behavioural Brain Research, 170, 156-162. https://doi.org/10.1016/j.bbr.2006.02.008.

Ohayon, M.M., Carskadon, M.A., Guilleminault, C., \& Vitiello, M.V. (2004). Meta-analysis of quantitative sleep parameters from childhood to old age in healthy individuals: Developing normative sleep values across the human lifespan. Sleep, 27, 1255-1273.

Owens, J. (2014). Insufficient sleep in adolescents and young adults: An update on causes and consequences. Pediatrics, 143(3), e921-e932. https://doi.org/10.1542/peds.2014-1696.

Owens, J.A., Belon, K., \& Moses, P. (2010). Impact of delaying school start time on adolescent sleep, mood, and behavior. Archives of Pediatrics \& Adolescent Medicine, 164(7), 608614. 
Ozawa, T., Yamada, K., \& Ichitani, Y. (2011). Long-term object location memory in rats: Effects of sample phase and delay length in spontaneous place recognition test. Neuroscience Letters, 497, 37-41. https://doi.org/10.1016/j.neulet.2011.04.022.

Palchykova, S., Crestani, F., Meerlo, P., \& Tobler, I. (2006). Sleep deprivation and daily torpor impair object recognition in Djungarian hamsters. Physiology \& Behavior, 87, 144-153. https://doi.org/10.1016/j.physbeh.2005.09.005.

Palchykova, S., Winsky-Sommerer, R., Meerlo, P., Dürr, R., \& Tobler, I. (2006). Sleep deprivation impairs object recognition in mice. Neurobiology of Learning and Memory, 85, 263-271. https://doi.org/10.1016/j.nim.2005.11.005.

Poe, G.R. (2017) Sleep is for forgetting. The Journal of Neuroscience, 37, 464-473. https://doi.org/10.1523/JNEUROSCI.0820-16.2017.

Ribeiro-Silva, N., Nejm, M.B., da Silva, S.M.A., Suchecki, D., \& Luz, J. (2016). Restriction of rapid eye movement sleep during adolescence increases energy gain and metabolic efficiency in young adult rats. Experimental Physiology, 101, 308-318. https://doi.org/10.1113/EP085323.

Riemann, D., Voderholzer, U., Spiegelhalder, K., Hornyak, M., Buysse, D.J., Nissen, C., . . Feige, B. (2007). Chronic insomnia and MRI-measured hippocampal volumes: A pilot study. Sleep, 30, 955-958.

Sapolsky, R.M. (2000). Glucocorticoids and hippocampal atrophy in neuropsychiatric disorders. Archives of General Psychiatry, 57, 925- 935.

Sara, S.J. (2017). Sleep to remember. The Journal of Neuroscience, 37, 457-463. https://doi.org/10.1177/1073858406292647. 
Saré, R.M., Levine, M., Hildreth, C., Picchioni, D., \& Smith, C.B. (2016). Chronic sleep restriction during development can lead to long-lasting behavioral effects. Physiology \& Behavior, 155, 208-217. https://doi.org/10.1016/j.physbeh.2015.12.019.

Schoenborn, C.A. \& Adams, P.F. (2010) Health behaviors of adults: United States, 2005-2007. Retrieved from https://www.cdc.gov/nchs/data/series/sr_10/sr10_245.pdf.

Shanks, N., Greek, R., \& Greek, J. (2009). Are animal models predictive for humans? Philosophy, Ethics, and Humanities in Medicine, 4(2). https://doi.org/10.1186/1747$5341-4-2$.

Silvers, J.M., Harrod, S.B., Mactutus, C.F., Booze, R.M. (2007). Automation of the novel object recognition tasks for use in adolescent rats. Journal of Neuroscience Methods, 166, 99103. https://doi.org/10.1016/j.jneumeth.2007.06.032.

Sisk, C.L. \& Zehr, J.L. (2005). Pubertal hormones organize the adolescent brain and behavior. Frontiers in Neuroendocrinology, 26, 163-174. https://doi.org/10.1016/j.yfrne.2005.10.003.

Smith, C.T., Conway, J.M., \& Rose, G.M. (1998). Brief paradoxical sleep deprivation impairs references, but not working, memory in the radial arm maze task. Neurobiology of Learning and Memory, 69, 211-217. https://doi.org/10.1006/nlme.1997.3809.

Smith, C. \& Smith, D. (2003). Ingestion of ethanol just prior to sleep onset impairs memory for procedural but not declarative tasks. Sleep, 26, 185-191.

Spear, L.P. (2000). The adolescent brain and age-related behavioral manifestations. Neuroscience \& Biobehavioral Reviews, 24, 417-463.

Spear, L.P. (2013). Adolescent neurodevelopment. Journal of Adolescent Health, 52, S7-S13. https://doi.org/10.1016/j.jadohealth.2012.05.006. 
Spear, L.P. (2015). Adolescent alcohol exposure: Are there separable vulnerable periods within adolescence? Physiology \& Behavior, 148, 122-130. https://doi.org/10.1016/j.physbeh.2015.01.027.

Spiegel, K., Knutson, K., Leproult, R., Tasali, E., \& Van Cauter, E. (2005). Sleep loss: A novel risk factor for insulin resistance and Type 2 diabetes. Journal of Applied Physiology, 99, 2008-2019. https://doi.org/10.1152/japplphysiol.00660.2005.

Stickgold, R., James, L., Hobson, J.A. (2000). Visual discrimination learning requires sleep after training. Nature, 3, 1237-1238. https://doi.org/10.1038/81756.

Strecker, T., Drinkenburg, W.H., Sahgal, A., \& Aggleton, J.P. (1998). Recognition memory in rats--I. Concepts and classifications. Progress in Neurobiology, 54, 289-311.

Tulving, E. \& Markowitsch, H.J. (1997). Memory beyond the hippocampus, Current Opinion in Neurobiology, 7, 209-216.

Vetter-O'Hagen, C.S. \& Spear, L.P. (2012). Hormonal and physical markers of puberty and their relationship to adolescent-type novelty-directed behavior. Developmental Pyschobiology, 54, 523-535. https://doi.org/10.1002/dev.20610.

Wahlstrom, K. (2002). First longitudinal study of later high school start times. NASSP Bulletin, $86(633), 3-21$.

Walker, M.P. (2010). Sleep, memory and emotion. Progress in Brain Research, 185, 49-68. https://doi.org/10.1016/B978-0-444-53702-7.00004-X

Walsh, C.M., Booth, V., \& Poe, G.R. (2011). Spatial and reversal learning in the Morris water maze are largely resistant to six hours of REM sleep deprivation following training. Learning \& Memory, 18, 422-434. https://doi.org/10.1101/lm.2099011. 
Ward, C.P., McCoy, J.G., McKenna, J.T., Connolly, N.P., McCarley, R.W., \& Strecker, R.E. (2009). Spatial learning and memory deficits following exposure to 24 hours of sleep fragmentation or intermittent hypoxia in a rat model of obstructive sleep apnea. Brain Research, 19, 128-137. https://doi.org/10.1016/j.brainres.2009.07.064.

Winters, B.D., Forwood, S.E., Cowell, R.A., Saksida, L.M., \& Bussey, T.J. (2004). Double dissociation between the effects of peri-postrhinal cortex and hippocampal lesions on tests of object recognition and spatial memory: heterogeneity of function within the temporal lobe. The Journal of Neuroscience, 26, 5901-5908. https://doi.org/10.1523/JNEUROSCI.1346-04.2004.

Yang, S., Sun, H., Huang, Z., Yao, M., \& Qu, W. (2012). Repeated sleep restriction in adolescent rats altered sleep patterns and impaired spatial learning/memory ability. Sleep, 35, 849-859A. https://doi.org/10.5665/sleep.1888.

Youngblood, B.D., Zhou, J., Smagin, G.N., Ryan, D.H., \& Harris, R.B.S. (1997). Sleep deprivation by the "flower pot" technique and spatial reference memory. Physiology \& Behavior, 61, 249-256. 
Appendix

\section{Seton Hall University \\ IACUC}

From: Michael Vigorito, Ph.D., Chair of IACUC

Date: $10 / 3 / 2017$

Re: IACUC Protocol AH1701 Approval

Your Protocol "The immediate and Long-lasting Cognitive Consequences of Adolescent Chronic Sleep Restriction" has been approved by the IACUC. It has been given the protocol number AH1701. The project start date is 10/3/2017 and expires 9/30/2018.

Also note that the IACUC must approve all changes to previously approved protocols prior to the change taking place. The Continuing Review and/or Modification Form (attached) can be used to obtain approval for a modification request.

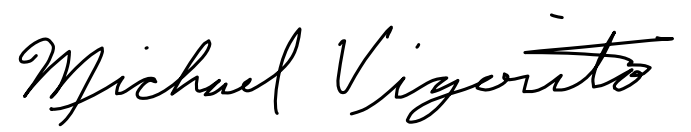

Michael Vigorito, Ph.D.

Professor

Department of Psychology

Seton Hall University

South Orange, NJ 07079

973-275-2707 (Office)

973-761-9484 (Department Office)

michael.vigorito@shu.edu 